\title{
Irreducible Hamiltonian BRST-anti-BRST symmetry for reducible systems
}

\author{
C. Bizdadea*, E. M. Cioroianu \\ Department of Physics, University of Craiova \\ 13 A. I. Cuza Str., Craiova RO-1100, Romania
}

December 14, 2018

\begin{abstract}
An irreducible Hamiltonian BRST-anti-BRST treatment of reducible first-class systems based on homological arguments is proposed. The general formalism is exemplified on the Freedman-Townsend model.
\end{abstract}

PACS number: 11.10.Ef

\section{Introduction}

The BRST formalism has been extended over the last few years to a more symmetrical approach, called the BRST-anti-BRST method. This type of extended BRST symmetry has been implemented at the Hamiltonian [1]-[6], as well as at the Lagrangian level [6]-[19]. Although it does not play such an important role like the BRST symmetry itself, the BRST-anti-BRST symmetry is, however, a useful tool in the geometric (superfield) description of the BRST transformation, for the perturbative investigation of the renormalizability of Yang-Mills theories, in a consistent approach to anomalies or for a correct understanding of the non-minimal sector from the BRST treatment

\footnotetext{
*e-mail addresses: bizdadea@central.ucv.ro and bizdadea@hotmail.com

$\dagger$ e-mail address: manache@central.ucv.ro

‡e-mail addresses: osaliu@central.ucv.ro
} 
[20]-[26]. The BRST-anti-BRST method can be equally used to investigate irreducible and reducible gauge theories or Hamiltonian systems possessing irreducible and reducible first-class constraints. Nevertheless, the Hamiltonian BRST-anti-BRST treatment of reducible first-class systems turns out to be a complicated mechanism, due, in principal, to the additional redundancy of the constraint functions implied by the inner structure of the BRST-antiBRST symmetry. This makes the computation of the total BRST charge and BRST-anti-BRST-invariant Hamiltonian, and especially of the gaugefixed action, a more difficult task than in the irreducible case.

In this paper we give an irreducible Hamiltonian BRST-anti-BRST treatment of on-shell reducible first-class theories that somehow simplifies the standard reducible approach. Our treatment is mainly based on the following steps: (a) we transform the original reducible first-class constraints into some irreducible ones on a larger phase-space in a manner that allows the substitution of the Hamiltonian BRST-anti-BRST symmetry of the reducible system by that of the irreducible theory; (b) we quantize the irreducible system accordingly with the Hamiltonian BRST-anti-BRST formalism. This programme will lead to the removal of all ghosts for ghosts and antighosts associated with the original reducibility. The idea of replacing a redundant theory with an irreducible one is not new [1], [27], and has recently been developed at the antifield BRST level for both Lagrangian gauge theories and Hamiltonian first-class systems [28]. The same idea has also been approached within the framework of the Lagrangian BRST-anti-BRST context [29], but it has neither been consistently developed, nor yet applied to the Hamiltonian BRST-anti-BRST approach to on-shell reducible first-class systems, hence our paper establishes a new result.

The paper is organized into six sections. Section 2 realizes a brief review of the Hamiltonian BRST-anti-BRST formalism for reducible first-class systems. Section 3 is focused on the derivation of an irreducible Koszul-Tate bicomplex associated with a reducible theory. This Koszul-Tate bicomplex is inferred from the requirement that all nontrivial co-cycles at total resolution degree one from the reducible approach exclusively due to the original redundancy become trivial under a proper redefinition of the antighosts with total resolution degree equal to one. This will underline an irreducible firstclass constraint set corresponding to the initial reducible one. Section 4 is concerned with the construction of the Hamiltonian BRST-anti-BRST symmetry of the irreducible theory. In the meantime, we establish the link with 
the reducible BRST-anti-BRST symmetry and show that it is permissible to replace the Hamiltonian BRST-anti-BRST symmetry of the initial reducible system with that of the irreducible theory. In section 5 we apply the theoretical part of the paper to the Freedman-Townsend model. Section 6 ends the paper with the main conclusions.

\section{Hamiltonian BRST-anti-BRST symmetry for reducible first-class systems}

In this section we give a brief review of the Hamiltonian BRST-anti-BRST symmetry for reducible first-class systems. Our starting point is a Hamiltonian system on a phase-space described locally by $N$ bosonic co-ordinates $z^{A}$, subject to the first-class constraints

$$
\Sigma: G_{a_{0}}\left(z^{A}\right) \approx 0, a_{0}=1, \cdots, M_{0}
$$

which are assumed to be on-shell $L$-stage reducible. We presume that the second-class constraints (if any) have been eliminated by means of the Dirac bracket. The first-class behaviour of the constraints is translated into

$$
\left[G_{a_{0}}, G_{b_{0}}\right]=C_{a_{0} b_{0}}^{c_{0}} G_{c_{0}},
$$

while the on-shell redundancy is written as

$$
\begin{gathered}
Z_{a_{1}}^{a_{0}} G_{a_{0}}=0, a_{1}=1, \cdots, M_{1}, \\
Z_{a_{2}}^{a_{1}} Z_{a_{1}}^{a_{0}}=C_{a_{2}}^{a_{0} b_{0}} G_{b_{0}}, a_{2}=1, \cdots, M_{2}, \\
\vdots \\
Z_{a_{L}}^{a_{L-1}} Z_{a_{L-1}}^{a_{L-2}}=C_{a_{L}}^{a_{L-2} b_{0}} G_{b_{0}}, a_{L}=1, \cdots, M_{L},
\end{gathered}
$$

where the symbol [,] denotes either the Poisson bracket, or the Dirac bracket if any second-class constraints were present. All the functions in (2-5) may involve the phase-space coordinates, while the coefficients $C_{a_{2}}^{a_{0} b_{0}}$ and the firstorder structure functions $C^{c_{0}} a_{0} b_{0}$ are antisymmetric in the upper, respectively, lower indices. The analysis is performed in the bosonic case, but can be easily extended to fermions by introducing some appropriate sign factors. 
The Hamiltonian BRST-anti-BRST symmetry for such a reducible system is given by two anticommuting differentials

$$
s_{R a} s_{R b}+s_{R b} s_{R a}=0, a, b=1,2,
$$

that can be made to split as

$$
s_{R a}=\delta_{R a}+D_{R a}+\cdots, a=1,2 .
$$

The operators $\left(\delta_{R a}\right)_{a=1,2}$ generate the Koszul-Tate differential bicomplex, which is bigraded accordingly the resolution bidegree bires $=\left(\right.$ res $_{1}$, res $\left._{2}\right)$, and furnishes an homological biresolution of smooth functions defined on the first-class surface (四), $C^{\infty}(\Sigma) . \quad\left(D_{R a}\right)_{a=1,2}$ are known as longitudinal exterior derivatives and provide the extended longitudinal bicomplex bigraded via the form bidegree biform $=\left(\right.$ form $_{1}$, form $\left.{ }_{2}\right)$, which offers a redundant description of the tangent space to the gauge orbits. We set, as usual, bires $\left(\delta_{R 1}\right)=(-1,0)$, bires $\left(\delta_{R 2}\right)=(0,-1)$, biform $\left(D_{R 1}\right)=(1,0)$, biform $\left(D_{R 2}\right)=(0,1)$, bires $\left(D_{R 1}\right)=(0,0)$, bires $\left(D_{R 2}\right)=(0,0)$. The remaining terms in $\left(s_{R a}\right)_{a=1,2}$, generically denoted by "...", are required in order to ensure the BRST-anti-BRST algebra defining relations (6). The generators of the Koszul-Tate bicomplex are known as antighosts, while those from the exterior longitudinal bicomplex are called ghosts. The operators $s_{R 1}$ and $s_{R 2}$ constitute the basic ingredients of the BRST-anti-BRST differential bicomplex, with the bigrading given by the new ghost bidegree bingh $=\left(n g h_{1}, n g h_{2}\right)$ defined like

$$
\left(n g h_{1}, n g h_{2}\right)=\left(\text { form }_{1}-\text { res }_{1}, \text { form }_{2}-\text { res }_{2}\right) \text {, }
$$

which is such that bingh $\left(s_{R 1}\right)=(1,0)$ and bingh $\left(s_{R 2}\right)=(0,1)$. Consequently, we have that bingh $\left(\delta_{R 1}\right)=(1,0)$, bingh $\left(\delta_{R 2}\right)=(0,1)$, bingh $\left(D_{R 1}\right)=$ $(1,0)$, bingh $\left(D_{R 2}\right)=(0,1)$. The crucial property of this double complex is that the zeroth order cohomology spaces of $s_{R 1}$ and $s_{R 2}$ are isomorphic to the algebra of physical observables (the algebra of gauge invariant functions defined on (11) ). Finally, a word on the relationship between the BRST-antiBRST and BRST symmetries. It has been shown [5] that the Hamiltonian BRST-anti-BRST symmetry for an arbitrary Hamiltonian first-class system exists provided the standard Hamiltonian BRST symmetry for the system can be properly constructed. Actually, the sum between the BRST and 
anti-BRST operators (total BRST transformation)

$$
s_{R}=s_{R 1}+s_{R 2},
$$

defines a simple BRST complex associated with a complete description of the first-class surface (1) obtained by doubling the first-class constraints, which is graded accordingly as the new ghost number $n g h=n g h_{1}+n g h_{2}$ $\left(n g h\left(s_{R}\right)=1\right)$. Accordingly, we have that the total BRST symmetry splits in the usual way as

$$
s_{R}=\delta_{R}+D_{R}+\cdots
$$

where

$$
\delta_{R}=\delta_{R 1}+\delta_{R 2},
$$

gives a simple Koszul-Tate complex graded by the total resolution degree res $=\operatorname{res}_{1}+\operatorname{res}_{2}\left(\operatorname{res}\left(\delta_{R}\right)=-1\right)$, that realizes a homological resolution of smooth functions defined on (1) corresponding to the redundant description of this first-class surface, the operator

$$
D_{R}=D_{R 1}+D_{R 2}
$$

leads to a simple exterior longitudinal complex graded by the total form degree form $=$ form $_{1}+$ form f $_{2}\left(\right.$ orm $\left(D_{R}\right)=1$, res $\left.\left(D_{R}\right)=0\right)$, which offers a redundant description of the tangent space to the gauge orbits, and the rest of the terms "..." ensure the nilpotency of $s_{R}$

$$
s_{R}^{2}=0 \text {. }
$$

Of course, the zeroth order cohomology space of the total BRST transformation is again isomorphic to the algebra of physical observables

$$
H^{0}\left(s_{R}\right) \simeq\{\text { physical observables }\} .
$$

The link between the new ghost bidegree and the usual ghost number $(g h)$ from the standard Hamiltonian BRST formalism is expressed by $g h=n g h_{1}-$ $n g h_{2}$.

After this brief review on the main ideas underlying the Hamiltonian BRST-anti-BRST formalism, we analyse the basic steps in the construction of the Koszul-Tate bicomplex, which should be performed in such a way 
to comply with the essential requirements, which are the nilpotency and anticommutivity of $\left(\delta_{R a}\right)_{a=1,2}$

$$
\delta_{R a} \delta_{R b}+\delta_{R b} \delta_{R a}=0, a, b=1,2,
$$

together with the conditions that define the biresolution property

$$
\begin{gathered}
H_{0,0}\left(\delta_{R a}\right)=C^{\infty}(\Sigma), a=1,2, \\
H_{j, k}\left(\delta_{R a}\right)=0, j, k \geq 0, j+k \neq 0, a=1,2,
\end{gathered}
$$

where $H_{j, k}\left(\delta_{R a}\right)$ denotes the space of elements with the resolution bidegree equal to $(j, k)$ that are $\delta_{R a}$-closed modulo $\delta_{R a}$-exact (in the following we will suggestively refer to (17) as the 'biacyclicity conditions'). In the case of a first-stage reducible Hamiltonian system $(L=1)$, subject to the first-class constraints (1) and the reducibility relations (3), we introduce the antighost spectrum [5]

$$
\begin{aligned}
\left(\stackrel{[1,0]}{\mathcal{P}}_{1 a_{0}}, \stackrel{[0,1]}{\mathcal{P}}_{2 a_{0}}\right) & ,\left(\stackrel{[2,0]}{P}_{1 a_{1}}, \stackrel{[1,1]}{P}_{2 a_{1}}, \stackrel{[0,2]}{P}_{3 a_{1}}, \stackrel{[1,1]}{\lambda}_{a_{0}}\right), \\
& \left(\stackrel{[2,1]}{\rho}_{1 a_{1}}, \stackrel{[1,2]}{\rho}_{2 a_{1}}\right),
\end{aligned}
$$

where $\stackrel{[m, n]}{F}$ signifies an object with bires $(F)=(m, n)$. The relation between bingh and bires in the case of all variables from the antighost spectrum is bingh $=\left(-r e s_{1},-r e s_{2}\right)$. The proper definitions of $\left(\delta_{R a}\right)_{a=1,2}$ acting on the generators from the Koszul-Tate bicomplex are given by

$$
\begin{gathered}
\delta_{R 1} \stackrel{[0,0]^{A}}{z}=0, \delta_{R 2} \stackrel{[0,0]}{z}^{A}=0, \\
\delta_{R 1} \stackrel{[1,0]}{\mathcal{P}}_{1 a_{0}}=-G_{a_{0}}, \delta_{R 2} \stackrel{[1,0]}{\mathcal{P}}_{1 a_{0}}=0, \\
\delta_{R 1} \stackrel{[0,1]}{\mathcal{P}}_{2 a_{0}}=0, \delta_{R 2} \stackrel{[0,1]}{\mathcal{P}}_{2 a_{0}}=-G_{a_{0}}, \\
\delta_{R 1} \stackrel{[2,0]}{P}_{1 a_{1}}=-\stackrel{[1,0]}{\mathcal{P}}_{1 a_{0}} Z_{a_{1}}^{a_{0}}, \delta_{R 2} \stackrel{[2,0]}{P}{ }_{1 a_{1}}=0, \\
\delta_{R 1} \stackrel{[1,1]}{P}_{2 a_{1}}=\frac{1}{2} \stackrel{[0,1]}{\mathcal{P}}_{2 a_{0}} Z_{a_{1}}^{a_{0}}, \delta_{R 2} \stackrel{[1,1]}{P}_{2 a_{1}}=\frac{1}{2} \stackrel{[1,0]}{\mathcal{P}}_{1 a_{0}} Z_{a_{1}}^{a_{0}},
\end{gathered}
$$




$$
\begin{gathered}
\delta_{R 1} \stackrel{[0,2]}{P}_{3 a_{1}}=0, \delta_{R 2} \stackrel{[0,2]}{P}_{3 a_{1}}=-\stackrel{[0,1]}{\mathcal{P}}_{2 a_{0}} Z_{a_{1}}^{a_{0}}, \\
\delta_{R 1} \stackrel{[1,1]}{\lambda}_{a_{0}}=-\stackrel{[0,1]}{\mathcal{P}}_{2 a_{0}}, \delta_{R 2} \stackrel{[1,1]}{\lambda}_{a_{0}}=\stackrel{[1,0]}{\mathcal{P}}_{1 a_{0}}, \\
\delta_{R 1} \stackrel{[}{[2,1]}_{1 a_{1}}=-\stackrel{[1,1]}{P}_{2 a_{1}}-\frac{1}{2} \stackrel{[1,1]}{\lambda}_{a_{0}} Z_{a_{1}}^{a_{0}}, \delta_{R 2} \stackrel{[}{\rho}_{1 a_{1}}=-\stackrel{[2,0]}{P}_{1 a_{1}}, \\
\delta_{R 1} \stackrel{[1,2]}{\rho}_{2 a_{1}}=-\stackrel{[0,2]}{P}_{3 a_{1}}, \delta_{R 2} \stackrel{[1,2]}{\rho}_{2 a_{1}}=-\stackrel{[1,1]}{P}_{2 a_{1}}+\frac{1}{2} \stackrel{[1,1]}{\lambda}_{a_{0}} Z_{a_{1}}^{a_{0}},
\end{gathered}
$$

and ensure the basic conditions (15 (17). For a second-stage first-class Hamiltonian theory $(L=2)$ described by the constraints (1) and the reducibility relations (3 - 4 ), one needs to supplement the antighost spectrum (18-19) with the antighosts

$$
\left(\stackrel{[3,0]}{P}_{1 a_{2}}, \stackrel{[2,1]}{P}_{2 a_{2}}, \stackrel{[1,2]}{P}_{3 a_{2}}, \stackrel{[0,3]}{P}_{4 a_{2}}\right),\left(\stackrel{[3,1]}{\rho}_{1 a_{2}}, \stackrel{[2,2]}{\rho}_{2 a_{2}}, \stackrel{[1,3]}{\rho}_{3 a_{2}}\right),
$$

and set the definitions (20 28) together with

$$
\begin{aligned}
& \delta_{R 1} \stackrel{[3,0]}{P}_{1 a_{2}}=-\stackrel{[2,0]}{P}_{1 a_{1}} Z_{a_{2}}^{a_{1}}+\frac{1}{2} C_{a_{2}}^{a_{0} b_{0}} \stackrel{[1,0]}{\mathcal{P}}_{1 a_{0}} \stackrel{[1,0]}{\mathcal{P}}_{1 b_{0}}, \delta_{R 2} \stackrel{[3,0]}{P}_{1 a_{2}}=0 \\
& \delta_{R 1} \stackrel{[2,1]}{P}{ }_{2 a_{2}}=\frac{1}{2} \stackrel{[1,1]}{P}_{2 a_{1}} Z_{a_{2}}^{a_{1}}+\frac{1}{4} C_{a_{2}}^{a_{0} b_{0}} \stackrel{[1,0]}{\mathcal{P}}_{1 a_{0}} \stackrel{[0,1]}{\mathcal{P}}_{2 b_{0}}, \\
& \delta_{R 2} \stackrel{[2,1]}{P}_{2 a_{2}}=-\frac{1}{2} \stackrel{[2,0]}{P}_{1 a_{1}} Z_{a_{2}}^{a_{1}}+\frac{1}{4} C_{a_{2}}^{a_{0} b_{0}} \stackrel{[1,0]}{\mathcal{P}}_{1 a_{0}} \stackrel{[1,0]}{\mathcal{P}}_{1 b_{0}}, \\
& \delta_{R 1} \stackrel{[1,2]}{P}_{3 a_{2}}=\frac{1}{2} \stackrel{[0,2]}{P}_{3 a_{1}} Z_{a_{2}}^{a_{1}}-\frac{1}{4} C_{a_{2}}^{a_{0} b_{0}} \stackrel{[0,1]}{\mathcal{P}}_{2 a_{0}} \stackrel{[0,1]}{\mathcal{P}}_{2 b_{0}}, \\
& \delta_{R 2} \stackrel{[1,2]}{P}_{3 a_{2}}=-\frac{1}{2} \stackrel{[1,1]}{P}_{2 a_{1}} Z_{a_{2}}^{a_{1}}-\frac{1}{4} C_{a_{2}}^{a_{0} b_{0}} \stackrel{[1,0]}{\mathcal{P}}_{1 a_{0}} \stackrel{[0,1]}{\mathcal{P}}_{2 b_{0}}, \\
& \delta_{R 1} \stackrel{[0,3]}{P}_{4 a_{2}}=0, \delta_{R 2} \stackrel{[0,3]}{P}_{4 a_{2}}=-\stackrel{[0,2]}{P}_{3 a_{1}} Z_{a_{2}}^{a_{1}}+\frac{1}{2} C_{a_{2}}^{a_{0} b_{0}} \stackrel{[0,1]}{\mathcal{P}}_{2 a_{0}} \stackrel{[0,1]}{\mathcal{P}}_{2 b_{0}}, \\
& \delta_{R 1} \stackrel{[3,1]}{\rho}_{1 a_{2}}=-\stackrel{[2,1]}{P}_{2 a_{2}}-\frac{1}{2} \stackrel{[2,1]}{\rho}_{1 a_{1}} Z_{a_{2}}^{a_{1}}+\frac{1}{4} C_{a_{2}}^{a_{0} b_{0}} \stackrel{[1,1]}{\lambda}_{a_{0}} \stackrel{[1,0]}{\mathcal{P}}_{1 b_{0}}, \\
& \delta_{R 2} \stackrel{[3,1]}{\rho}_{1 a_{2}}=\stackrel{[3,0]}{P}_{1 a_{2}}, \\
& \delta_{R 1} \stackrel{[2,2]}{\rho}_{2 a_{2}}=-\stackrel{[1,2]}{P}_{3 a_{2}}-\frac{1}{2} \stackrel{[1,2]}{\rho}_{2 a_{1}} Z_{a_{2}}^{a_{1}}-\frac{1}{4} C_{a_{2}}^{a_{0} b_{0}} \stackrel{[1,1]}{\lambda}_{a_{0}} \stackrel{[0,1]}{\mathcal{P}}_{2 b_{0}},
\end{aligned}
$$




$$
\begin{gathered}
\delta_{R 2} \stackrel{[2,2]}{\rho}_{2 a_{2}}=-\stackrel{[2,1]}{P}_{2 a_{2}}+\frac{1}{2} \stackrel{[2,1]}{\rho}_{1 a_{1}} Z_{a_{2}}^{a_{1}}-\frac{1}{4} C_{a_{2}}^{a_{0} b_{0}} \stackrel{[1,1]}{\lambda}_{a_{0}} \stackrel{[1,0]}{\mathcal{P}}_{1 b_{0}}, \\
\delta_{R 1} \stackrel{[1,3]}{\rho}_{3 a_{2}}=-\stackrel{[0,3]}{P}_{4 a_{2}}, \\
\delta_{R 2} \stackrel{[1,3]}{\rho}_{3 a_{2}}=-\stackrel{[1,2]}{P}_{3 a_{2}}+\frac{1}{2} \stackrel{[1,2]}{\rho}_{2 a_{1}} Z_{a_{2}}^{a_{1}}+\frac{1}{4} C_{a_{2}}^{a_{0} b_{0}} \stackrel{[1,1]}{\lambda}_{a_{0}}^{[0,1]}{ }_{2 b_{0}},
\end{gathered}
$$

in order to obtain (15 17). Similarly, in the general case of an $L$-stage reducible Hamiltonian system possessing the first-class constraints (11) and subject to the reducibility relations (3-5), the Koszul-Tate bicomplex includes the generators

$$
\begin{aligned}
& \left(\stackrel{[1,0]}{\mathcal{P}}_{1 a_{0}}, \stackrel{[0,1]}{\mathcal{P}}_{2 a_{0}}\right), \\
& \left(\stackrel{[2,0]}{P}_{1 a_{1}}, \stackrel{[1,1]}{P}_{2 a_{1}}, \stackrel{[0,2]}{P}_{3 a_{1}} \stackrel{[1,1]}{\lambda}_{a_{0}}\right),
\end{aligned}
$$

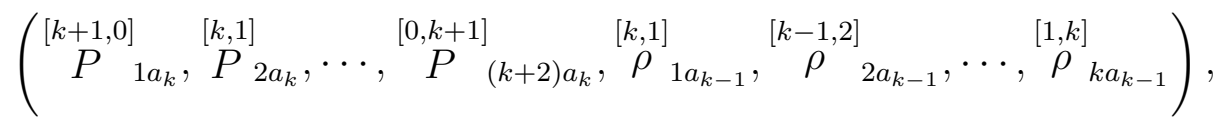

$$
\begin{aligned}
& \left(\begin{array}{c}
{[L+1,1]} \\
\rho
\end{array} 1 a_{L}, \stackrel{[L, 2]}{\rho}_{2 a_{L}}, \cdots, \stackrel{[1, L+1]}{\rho}_{(L+1) a_{L}}\right)
\end{aligned}
$$

with $k=2, \cdots, L$, on which $\left(\delta_{R a}\right)_{a=1,2}$ act in a way that complies with 15 17). We note that the antighosts (42 45) are organized on levels of increasing total resolution degree, all the antighosts in say (44) displaying the same resolution degree, $k+1$.

\section{Irreducible Koszul-Tate bicomplex}

Here, we investigate the possibility of associating an irreducible Koszul-Tate bicomplex with the initial reducible one. By 'irreducible Koszul-Tate bicomplex' we understand a double complex underlying some irreducible first-class constraints, and hence whose redundancy is dictated only by the reducibility relations resulting from the doubling of constraint functions. The cornerstone of our approach relies on redefining the antighosts (42) of total resolution degree one such that all the co-cycles of $\left(\delta_{R a}\right)_{a=1,2}$ at total resolution degree one due exclusively to the original reducibility relations (35) become trivial 
(either identically vanish or are made exact). Then, all the corresponding higher-order resolution degree antighosts will be eliminated from the antighost spectrum, which will retain only the generators $\stackrel{[1,0]}{\mathcal{P}}_{1 a_{0}}, \stackrel{[0,1]}{\mathcal{P}}_{2 a_{0}}$ and $[1,1]$

$\lambda_{a_{0}}$ from the reducible treatment. Consequently, we expect the phase-space and antighost spectrum to be modified in order to furnish a biresolution of smooth functions defined on the surface of irreducible first-class constraints, but only up to some new bosonic canonical pairs, respectively, antighosts of resolution bidegrees $(1,0),(0,1)$ or $(1,1)$. For the sake of clarity, we initially analyse the cases $L=1,2$, and then generalize the results to an arbitrary $L$.

\subsection{First-stage reducible theories}

We begin with a first-stage reducible set of first-class constraints $(L=1)$, described by the formulae (1-3). The reducible Koszul-Tate bicomplex is then fully determined by the generators (18 19), and the definitions (20 28). The co-cycles of $\left(\delta_{R a}\right)_{a=1,2}$ at total resolution degree one caused by the initial redundancy relations (3) are obviously given by

$$
\mu_{a_{1}}^{1}=\stackrel{[1,0]}{\mathcal{P}}_{1 a_{0}} Z_{a_{1}}^{a_{0}}, \mu_{a_{1}}^{2}=\stackrel{[0,1]}{\mathcal{P}}_{2 a_{0}} Z_{a_{1}}^{a_{0}} .
$$

Our main concern is to investigate whether or not it is possible to perform a transformation of the type

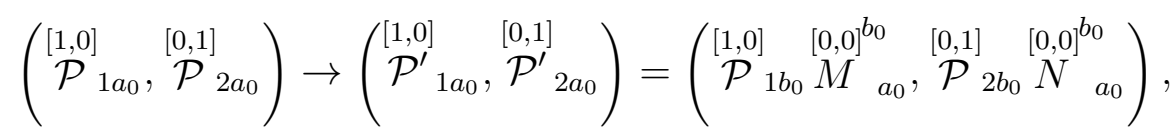

that makes the new co-cycles of the type (46) trivial. The above redefinition of the antighosts is such as to preserve the original resolution bidegrees. Thus, the matrices $M$ and $N$ can depend at most on the phase-space variables, $z^{A}$. Taking into account the symmetry between the BRST and anti-BRST components present everywhere in the development of the BRST-anti-BRST formalism, it is natural to take

$$
\stackrel{[0,0]}{M}_{a_{0}}^{b_{0}}=\stackrel{[0,0}{N}_{a_{0}}^{b_{0}} \equiv T_{a_{0}}^{b_{0}} .
$$

If we impose on the matrix $T_{a_{0}}^{b_{0}}$ the conditions

$$
T_{a_{0}}^{b_{0}} G_{b_{0}}=G_{a_{0}}, T_{a_{0}}^{b_{0}} Z_{a_{1}}^{a_{0}} \equiv 0
$$


and multiply the former definitions in (21) and the latter ones in (22) by $T_{a_{0}}^{b_{0}}$, we obtain

$$
\delta_{1} \stackrel{[1,0]}{\mathcal{P}}_{1 a_{0}}^{\prime}=-G_{a_{0}}, \delta_{2}{\stackrel{[0,1]}{\mathcal{P}^{\prime}}}_{2 a_{0}}=-G_{a_{0}} .
$$

The formulae (50) will lead to some co-cycles of the type (46), i.e.,

$$
\mu_{a_{1}}^{\prime 1}=\stackrel{[1,0]}{\mathcal{P}^{\prime}}{ }_{1 a_{0}} Z_{a_{1}}^{a_{0}}, \mu_{a_{1}}^{\prime 2}=\stackrel{[0,1]}{\mathcal{P}^{\prime}}{ }_{2 a_{0}} Z_{a_{1}}^{a_{0}},
$$

that are found to be trivial $\left(\mu_{a_{1}}^{\prime 1} \equiv 0, \mu_{a_{1}}^{\prime 2} \equiv 0\right)$ thanks to the latter condition in (49). In this way, it results that the co-cycles (51) are trivial as long as equations (49) possess solutions. The solution to (49) is

$$
T_{a_{0}}^{b_{0}}=\delta_{a_{0}}^{b_{0}}-Z_{b_{1}}^{b_{0}} \bar{D}_{a_{1}}^{b_{1}} A_{a_{0}}^{a_{1}}
$$

where the functions $A_{a_{0}}^{a_{1}}$ can at most involve $z^{A}$ and are taken to satisfy

$$
\operatorname{rank}\left(Z_{a_{1}}^{a_{0}} A_{a_{0}}^{b_{1}}\right) \equiv \operatorname{rank}\left(D_{a_{1}}^{b_{1}}\right)=M_{1}
$$

with $\bar{D}_{a_{1}}^{b_{1}}$ denoting the inverse of $D_{a_{1}}^{b_{1}}=Z_{a_{1}}^{a_{0}} A_{a_{0}}^{b_{1}}$. Furthermore, as $\mu_{a_{1}}^{\prime 1}$ and $\mu_{a_{1}}^{\prime 2}$ are no longer co-cycles, the antighosts $\stackrel{[2,0]}{P}_{1 a_{1}}, \stackrel{[1,1]}{P}_{2 a_{1}}, \stackrel{[0,2]}{P}_{3 a_{1}}$ will be discarded from the antighost spectrum (18), which consequently implies the removal of $\stackrel{[2,1]}{\rho}_{1 a_{1}}, \stackrel{[1,2]}{\rho}_{2 a_{1}}$ from (19). In order to outline the irreducibility of the resulting Koszul-Tate bicomplex, in (50) we employed the notation $\delta_{a}$ instead of $\delta_{R a}$. Obviously, the complete actions of the BRST and anti-BRST Koszul-Tate operators on the new generators $\stackrel{[1,0]}{\mathcal{P}}_{1 a_{0}}$ and $\stackrel{[0,1]}{\mathcal{P}}_{2 a_{0}}$ are realized as

$$
\begin{gathered}
\delta_{1}{\stackrel{[1,0]}{\mathcal{P}^{\prime}}}_{1 a_{0}}=-G_{a_{0}}, \delta_{2}{\stackrel{[1,0]}{\mathcal{P}^{\prime}}}_{1 a_{0}}=0, \\
\delta_{1}{\stackrel{[0,1]}{\mathcal{P}^{\prime}}}_{2 a_{0}}=0, \delta_{2}{\stackrel{[0,1]}{\mathcal{P}^{\prime}}}_{2 a_{0}}=-G_{a_{0}} .
\end{gathered}
$$

Inserting the relations (47) and (52) in formulae (54 55), we deduce that (54-55) are equivalent to

$$
\delta_{1} \stackrel{[1,0]}{\mathcal{P}}_{1 a_{0}}=-G_{a_{0}}+\delta_{1}\left(A_{a_{0}}^{a_{1}} \bar{D}_{a_{1}}^{b_{1}} Z_{b_{1}}^{b_{0}} \stackrel{[1,0]}{\mathcal{P}}_{1 b_{0}}\right), \delta_{2} \stackrel{[1,0]}{\mathcal{P}}_{1 a_{0}}=0
$$




$$
\delta_{1} \stackrel{[0,1]}{\mathcal{P}}_{2 a_{0}}=0, \delta_{2} \stackrel{[0,1]}{\mathcal{P}}_{2 a_{0}}=-G_{a_{0}}+\delta_{2}\left(A_{a_{0}}^{a_{1}} \bar{D}_{a_{1}}^{b_{1}} Z_{b_{1}}^{b_{0}} \stackrel{[0,1]}{\mathcal{P}}_{2 b_{0}}\right) .
$$

Our next concern is to determine the structure of the irreducible first-class constraints constituting the core of this irreducible bicomplex. In this light, we enlarge the phase-space by adding some new bosonic canonical pairs $z^{A_{1}} \equiv$ $\left(y^{a_{1}}, \pi_{a_{1}}\right)$ with resolution bidegree $(0,0)$, whose momenta are restricted to be nonvanishing solutions to the equations

$$
\begin{aligned}
& D_{a_{1}}^{b_{1}} \pi_{b_{1}}=\delta_{1}\left(-Z_{a_{1}}^{b_{0}} \stackrel{[1,0]}{\mathcal{P}}_{1 b_{0}}\right), \\
& D_{a_{1}}^{b_{1}} \pi_{b_{1}}=\delta_{2}\left(-Z_{a_{1}}^{b_{0}} \stackrel{[0,1]}{\mathcal{P}}_{2 b_{0}}\right),
\end{aligned}
$$

and on which $\left(\delta_{a}\right)_{a=1,2}$ are set to act like

$$
\delta_{1} z^{A_{1}}=0, \delta_{2} z^{A_{1}}=0
$$

On account of the invertibility of $D^{b_{1}}{ }_{a_{1}}$, the nonvanishing solutions to the equations (58 59) ensure the irreducibility as these equations possess nonvanishing solutions if and only if (46) are no longer closed, and hence not co-cycles. Substituting (58 59) into (56-57), it follows that the actions of the irreducible Koszul-Tate differentials on the initial generators with total resolution degree one are given by

$$
\begin{gathered}
\delta_{1} \stackrel{[1,0]}{\mathcal{P}}_{1 a_{0}}=-G_{a_{0}}-A_{a_{0}}^{a_{1}} \pi_{a_{1}}, \delta_{2} \stackrel{[1,0]}{\mathcal{P}}_{1 a_{0}}=0, \\
\delta_{1} \stackrel{[0,1]}{\mathcal{P}}_{2 a_{0}}=0, \delta_{2} \stackrel{[0,1]}{\mathcal{P}}_{2 a_{0}}=-G_{a_{0}}-A_{a_{0}}^{a_{1}} \pi_{a_{1}},
\end{gathered}
$$

which offer us the concrete form of the searched for irreducible constraints like

$$
\gamma_{a_{0}} \equiv G_{a_{0}}+A_{a_{0}}^{a_{1}} \pi_{a_{1}} \approx 0 .
$$

The first-class behaviour of the new constraints results from (63), which, after some obvious computation, produce

$$
\pi_{a_{1}}=\bar{D}_{a_{1}}^{b_{1}} Z_{b_{1}}^{b_{0}} \gamma_{b_{0}}, G_{a_{0}}=\left(\delta_{a_{0}}^{b_{0}}-Z_{b_{1}}^{b_{0}} \bar{D}_{a_{1}}^{b_{1}} A_{a_{0}}^{a_{1}}\right) \gamma_{b_{0}},
$$


which consequently yield

$$
\left[\gamma_{a_{0}}, \gamma_{b_{0}}\right]=\bar{C}_{a_{0} b_{0}}^{c_{0}} \gamma_{c_{0}}
$$

for some $\bar{C}^{c_{0}} a_{0} b_{0}$.

In conclusion, we passed from the initial reducible Koszul-Tate bicomplex associated with the first-stage reducible first-class constraints (1) to an irreducible one, based on the generators

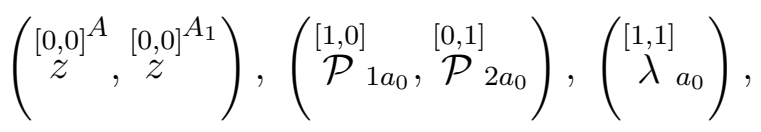

and the definitions

$$
\begin{gathered}
\delta_{1} z^{A}=0, \delta_{2} z^{A}=0, \delta_{1} z^{A_{1}}=0, \delta_{2} z^{A_{1}}=0, \\
\delta_{1} \stackrel{[1,0]}{\mathcal{P}}_{1 a_{0}}=-\gamma_{a_{0}}, \delta_{2} \stackrel{[1,0]}{\mathcal{P}}_{1 a_{0}}=0, \\
\delta_{1} \stackrel{[0,1]}{\mathcal{P}}_{2 a_{0}}=0, \delta_{2} \stackrel{[0,1]}{\mathcal{P}}_{2 a_{0}}=-\gamma_{a_{0}}, \\
\delta_{1} \stackrel{[1,1]}{\lambda}_{a_{0}}=-\stackrel{[0,1]}{\mathcal{P}}{ }_{2 a_{0}}, \delta_{2} \stackrel{[1,1]}{\lambda}_{a_{0}}=\stackrel{[1,0]}{\mathcal{P}}_{1 a_{0}},
\end{gathered}
$$

where the irreducible first-class constraint functions $\gamma_{a_{0}}$ are expressed by (63). It can be simply checked that the relations (67 70) define a correct Koszul-Tate bicomplex, that satisfies the basic requirements (15-17), with $\delta_{R a}$ and $C^{\infty}(\Sigma)$ replaced by $\delta_{a}$, respectively, $C^{\infty}\left(\Sigma^{\prime}\right)$, where $\Sigma^{\prime}: \gamma_{a_{0}} \approx 0$.

At this moment it is important to make two essential remarks. Firstly, the number of physical degrees of freedom is kept unchanged when passing to the irreducible setting. This is because in the reducible case there are $N$ canonical variables and $M_{0}-M_{1}$ independent first-class constraints, and hence $\frac{N}{2}-$ $M_{0}+M_{1}$ physical degrees of freedom, while in the irreducible situation there are $N+2 M_{1}$ canonical variables and $M_{0}$ independent first-class constraints, and therefore as many physical degrees of freedom as in the reducible version. Secondly, from (58 59) it results (due to the invertibility of $D^{b_{1}} a_{1}$ ) that the momenta $\pi_{a_{1}}$ are both $\delta_{1}$ - and $\delta_{2}$-exact. These observations represent two main conditions to also be imposed in connection with higher-order reducible Hamiltonian systems. Actually, we will see that for higher-order reducible theories it will be necessary to further add some bosonic canonical variables 
with the resolution bidegrees $(0,0)$ or $(1,1)$, and some fermionic antighosts of total resolution degree one. The former condition is directly correlated to the number of new canonical pairs and first-class constraints needed within the irreducible framework, while the latter implements the existence of a proper redefinition of the antighosts with total resolution degree one that renders trivial the co-cycles from the reducible approach.

\subsection{Second-stage reducible theories}

In this situation we start from an on-shell second-stage reducible set of firstclass constraints $(L=2)$, pictured by the formulae (1-4). The reducible Koszul-Tate bicomplex is now generated by (18 19) and (29). Obviously, the co-cycles of $\left(\delta_{R a}\right)_{a=1,2}$ at total resolution degree one exclusively due to the original reducibility relations (3- 44) are those appearing in the first-stage case, namely, (46). We are going to maintain the definitions (67 70), with $\gamma_{0}$ given precisely by (63). Still, we have to surpass two obstructions that prevent us from employing the analysis realized in the case $L=1$. Firstly, the matrix $D^{b_{1}}{ }_{a_{1}}=Z_{a_{1}}^{a_{0}} A_{a_{0}}^{b_{1}}$ is no longer invertible, so formula (52) is meaningless, such that the transformations (47) are inadequate. This is because $D^{b_{1}}{ }_{a_{1}}$ displays on-shell null vectors

$$
D_{a_{1}}^{b_{1}} Z_{a_{2}}^{a_{1}}=A_{a_{0}}^{b_{1}} Z_{a_{1}}^{a_{0}} Z_{a_{2}}^{a_{1}}=A_{a_{0}}^{b_{1}} C_{a_{2}}^{a_{0} b_{0}} G_{b_{0}} \approx 0
$$

hence now we have that $\operatorname{rank}\left(D_{a_{1}}^{b_{1}}\right)=M_{1}-M_{2}$. Under these circumstances, we will see that there is, however, possible to perform an appropriate redefinition of the antighosts $\stackrel{[1,0]}{\mathcal{P}} 1 a_{0}$ and $\stackrel{[0,1]}{\mathcal{P}}_{2 a_{0}}$ that brings the constraint functions $\gamma_{a_{0}}$ to the form (63) and, in the meantime, restores the triviality of the cocycles of the type (46). Secondly, we observe that the irreducible constraint functions (63) cannot ensure a number of physical degrees of freedom in the irreducible framework equal with the initial one. Rather, they should be supplemented with $M_{2}$ new constraints $\gamma_{a_{2}} \approx 0$ such that the entire constraint set is irreducible and first-class. Accordingly, we have to enrich the resulting irreducible Koszul-Tate bicomplex with the new antighosts $\stackrel{[1,0]}{\mathcal{P}}_{1 a_{2}}, \stackrel{[0,1]}{\mathcal{P}}_{2 a_{2}}$ and $[1,1]$

$\stackrel{[1,}{\lambda}_{a_{2}}$ and set the corresponding definitions

$$
\delta_{1} \stackrel{[1,0]}{\mathcal{P}}_{1 a_{2}}=-\gamma_{a_{2}}, \delta_{2} \stackrel{[1,0]}{\mathcal{P}}_{1 a_{2}}=0
$$




$$
\begin{gathered}
\delta_{1} \stackrel{[0,1]}{\mathcal{P}}_{2 a_{2}}=0, \delta_{2} \stackrel{[0,1]}{\mathcal{P}}_{2 a_{2}}=-\gamma_{a_{2}}, \\
\delta_{1} \stackrel{[1,1]}{\lambda}_{a_{2}}=-\stackrel{[0,1]}{\mathcal{P}}_{2 a_{2}}, \delta_{2} \stackrel{[1,1]}{\lambda}_{a_{2}}=\stackrel{[1,0]}{\mathcal{P}}_{1 a_{2}} .
\end{gathered}
$$

The appearance of the antighosts $\stackrel{[1,1]}{\lambda}_{a_{2}}$ is dictated by the latter and former definitions in (72), respectively, (73), and is strictly demanded by the supplementary trivial redundancy in the Hamiltonian BRST-anti-BRST formalism due to the doubling of the constraint functions. In brief, our programme in the case $L=2$ consists in determining some $\gamma_{a_{2}}$ that restore a correct irreducible Koszul-Tate bicomplex relying on the definitions (67 70) and (72 74), and in further finding a transformation of the antighosts $\stackrel{[1,0]}{\mathcal{P}}_{1 a_{0}}, \stackrel{[0,1]}{\mathcal{P}}_{2 a_{0}}$ (eventually involving the additional antighosts) that leads to some trivial co-cycles of the type (46) and, at the same time, is in agreement with (68 69).

In order to solve the former problem, we remark that condition (71) enables us to represent $D_{a_{1}}^{b_{1}}$ as

$$
D_{a_{1}}^{b_{1}}=\delta_{a_{1}}^{b_{1}}-Z_{b_{2}}^{b_{1}} \bar{D}_{a_{2}}^{b_{2}} A_{a_{1}}^{a_{2}}+A_{a_{0}}^{b_{1}} C_{c_{2}}^{a_{0} b_{0}} \bar{D}_{b_{2}}^{c_{2}} A_{a_{1}}^{b_{2}} G_{b_{0}}
$$

where $\bar{D}_{a_{2}}^{b_{2}}$ is the inverse of $D_{a_{2}}^{b_{2}}=Z_{a_{2}}^{c_{1}} A_{c_{1}}^{b_{2}}$ and $A_{c_{1}}^{b_{2}}$ are some functions that may depend at most on $z^{A}$ and are taken to fulfill $\operatorname{rank}\left(D_{b_{2}}^{a_{2}}\right)=M_{2}$. As we have previously stated, we maintain the definitions (68 69), with $\gamma_{a_{0}}$ as in (63). Then, if we apply $Z_{a_{1}}^{a_{0}}$ on these relations, we consequently find

$$
\begin{aligned}
& \delta_{1}\left(\stackrel{[1,0]}{\mathcal{P}}_{1 a_{0}} Z_{a_{1}}^{a_{0}}\right)=-\pi_{b_{1}} D_{a_{1}}^{b_{1}}, \\
& \delta_{2}\left(\stackrel{[0,1]}{\mathcal{P}}_{2 a_{0}} Z_{a_{1}}^{a_{0}}\right)=-\pi_{b_{1}} D_{a_{1}}^{b_{1}} .
\end{aligned}
$$

Taking into account the representation (75), formulae (76 77) become

$$
\begin{aligned}
& \delta_{1}\left(\stackrel{[1,0]}{\mathcal{P}}_{1 a_{0}} Z_{a_{1}}^{a_{0}}\right)=-\pi_{a_{1}}+\pi_{b_{1}} Z_{b_{2}}^{b_{1}} \bar{D}_{a_{2}}^{b_{2}} A_{a_{1}}^{a_{2}}-\pi_{b_{1}} A_{a_{0}}^{b_{1}} C_{c_{2}}^{a_{0} b_{0}} G_{b_{0}} \bar{D}_{b_{2}}^{c_{2}} A_{a_{1}}^{b_{2}}, \\
& \delta_{2}\left(\stackrel{[0,1]}{\mathcal{P}}_{2 a_{0}} Z_{a_{1}}^{a_{0}}\right)=-\pi_{a_{1}}+\pi_{b_{1}} Z_{b_{2}}^{b_{1}} \bar{D}_{a_{2}}^{b_{2}} A_{a_{1}}^{a_{2}}-\pi_{b_{1}} A_{a_{0}}^{b_{1}} C_{c_{2}}^{a_{0} b_{0}} G_{b_{0}} \bar{D}_{b_{2}}^{c_{2}} A_{a_{1}}^{b_{2}},
\end{aligned}
$$


which further yield

$$
\begin{aligned}
& \delta_{1}\left(\stackrel{[1,0]}{\mathcal{P}}_{1 a_{0}}\left(Z_{a_{1}}^{a_{0}}-C_{c_{2}}^{a_{0} b_{0}} G_{b_{0}} \bar{D}_{b_{2}}^{c_{2}} A_{a_{1}}^{b_{2}}\right)\right)=-\pi_{a_{1}}+\pi_{b_{1}} Z_{b_{2}}^{b_{1}} \bar{D}_{a_{2}}^{b_{2}} A_{a_{1}}^{a_{2}}, \\
& \delta_{2}\left(\stackrel{[0,1]}{\mathcal{P}}_{2 a_{0}}\left(Z_{a_{1}}^{a_{0}}-C_{c_{2}}^{a_{0} b_{0}} G_{b_{0}} \bar{D}_{b_{2}}^{c_{2}} A_{a_{1}}^{b_{2}}\right)\right)=-\pi_{a_{1}}+\pi_{b_{1}} Z_{b_{2}}^{b_{1}} \bar{D}_{a_{2}}^{b_{2}} A_{a_{1}}^{a_{2}} .
\end{aligned}
$$

The latter two relations are implied by $(78 \sqrt[79]{ })$ through the antisymmetry of $C_{c_{2}}^{a_{0} b_{0}}$ (from which it results that $\pi_{b_{1}} A_{a_{0}}^{b_{1}} C_{c_{2}}^{a_{0} b_{0}} G_{b_{0}}=\gamma_{a_{0}} C_{c_{2}}^{a_{0} b_{0}} G_{b_{0}}$ ) combined with formulae (67 69). The presence of the second term in the right-hand sides of (80 81) shows that the momenta $\pi_{a_{1}}$ are neither $\delta_{1^{-}}$, nor $\delta_{2}$-exact. The most natural choice to surpass this difficulty is to take the searched for functions $\gamma_{a_{2}}$ as

$$
\gamma_{a_{2}} \equiv \pi_{b_{1}} Z_{a_{2}}^{b_{1}}
$$

such that with the help of $(7273)$ we arrive at

$$
\delta_{1} \stackrel{[1,0]}{\mathcal{P}}_{1 a_{2}}=-\pi_{a_{1}} Z_{a_{2}}^{a_{1}}, \delta_{2} \stackrel{[0,1]}{\mathcal{P}}_{2 a_{2}}=-\pi_{a_{1}} Z_{a_{2}}^{a_{1}} .
$$

Accordingly, from (80 81) and (83) we recover the $\left(\delta_{a}\right)_{a=1,2}$-exactness of these momenta

$$
\begin{aligned}
& \pi_{a_{1}}=\delta_{1}\left(\stackrel{[1,0]}{\mathcal{P}}_{1 a_{0}}\left(-Z_{a_{1}}^{a_{0}}+C_{c_{2}}^{a_{0} b_{0}} G_{b_{0}} \bar{D}_{b_{2}}^{c_{2}} A_{a_{1}}^{b_{2}}\right)-\stackrel{[1,0]}{\mathcal{P}}_{1 b_{2}} \bar{D}_{a_{2}}^{b_{2}} A_{a_{1}}^{a_{2}}\right), \\
& \pi_{a_{1}}=\delta_{2}\left(\stackrel{[0,1]}{\mathcal{P}}_{2 a_{0}}\left(-Z_{a_{1}}^{a_{0}}+C_{c_{2}}^{a_{0} b_{0}} G_{b_{0}} \bar{D}_{b_{2}}^{c_{2}} A_{a_{1}}^{b_{2}}\right)-\stackrel{[0,1]}{\mathcal{P}}_{2 b_{2}} \bar{D}_{a_{2}}^{b_{2}} A_{a_{1}}^{a_{2}}\right) .
\end{aligned}
$$

In the meantime, as the functions $Z_{a_{2}}^{a_{1}}$ have no null vectors (the original set of constraints is by assumption second-stage reducible, so $Z_{a_{2}}^{a_{1}}$ are supposed to be independent), relations (83) provoke no nontrivial co-cycles. This solves the former problem set in the above in an appropriate manner.

Related to the latter problem (the existence of a transformation of the antighosts at total resolution degree one outputting some trivial co-cycles of the type (46) and being in accordance with (68 69)), we observe that replacing the relations (84 85) in the definitions (68), with $\gamma_{a_{0}}$ given by (63), it follows that

$$
\delta_{1} \stackrel{[1,0]}{\mathcal{P}}_{1 a_{0}}^{\prime \prime}=-G_{a_{0}}, \delta_{2} \stackrel{[0,1]}{\mathcal{P}}_{2 a_{0}}^{\prime \prime}=-G_{a_{0}}
$$


where

$$
\begin{aligned}
& \stackrel{[1,0]}{\mathcal{P}}^{\prime \prime}{ }_{1 a_{0}}=\stackrel{[1,0]}{\mathcal{P}}_{1 a_{0}}-\stackrel{[1,0]}{\mathcal{P}}_{1 b_{0}} Z_{b_{1}}^{b_{0}} A_{a_{0}}^{b_{1}}+ \\
& \stackrel{[1,0]}{\mathcal{P}}_{1 b_{0}} C_{c_{2}}^{b_{0} c_{0}} G_{c_{0}} \bar{D}_{b_{2}}^{c_{2}} A_{a_{1}}^{b_{2}} A_{a_{0}}^{a_{1}}-\stackrel{[1,0]}{\mathcal{P}}_{1 b_{2}} \bar{D}_{a_{2}}^{b_{2}} A_{a_{1}}^{a_{2}} A_{a_{0}}^{a_{1}}, \\
& \stackrel{[0,1]}{\mathcal{P}}_{2 a_{0}}=\stackrel{[0,1]}{\mathcal{P}}_{2 a_{0}}-\stackrel{[0,1]}{\mathcal{P}}_{2 b_{0}} Z_{b_{1}}^{b_{0}} A_{a_{0}}^{b_{1}}+ \\
& \stackrel{[0,1]}{\mathcal{P}}_{2 b_{0}} C_{c_{2}}^{b_{0} c_{0}} G_{c_{0}} \bar{D}_{b_{2}}^{c_{2}} A_{a_{1}}^{b_{2}} A_{a_{0}}^{a_{1}}-\stackrel{[0,1]}{\mathcal{P}}_{2 b_{2}} \bar{D}_{a_{2}}^{b_{2}} A_{a_{1}}^{a_{2}} A_{a_{0}}^{a_{1}} .
\end{aligned}
$$

After some computation, we gain the triviality of the new co-cycles

$$
\mu_{1 a_{1}}^{\prime \prime}=\stackrel{[1,0]}{\mathcal{P}^{\prime \prime}}{ }_{1 a_{0}} Z_{a_{1}}^{a_{0}}, \mu_{2 a_{1}}^{\prime \prime}=\stackrel{[0,1]}{\mathcal{P}^{\prime \prime}}{ }_{2 a_{0}} Z_{a_{1}}^{a_{0}},
$$

at total resolution degree one that result from (86), namely,

$$
\begin{aligned}
& \mu_{1 a_{1}}^{\prime \prime}=\delta_{1}\left(\frac{1}{2}{\stackrel{[1,0]}{\mathcal{P}^{\prime \prime}}}_{1 b_{0}} \stackrel{[1,0]}{\mathcal{P}^{\prime \prime}}{ }_{1 a_{0}} C_{c_{2}}^{a_{0} b_{0}} \bar{D}_{b_{2}}^{c_{2}} A_{a_{1}}^{b_{2}}\right) \\
& \mu_{2 a_{1}}^{\prime \prime}=\delta_{2}\left(\frac{1}{2} \stackrel{[0,1]}{\mathcal{P}^{\prime \prime}}{ }_{2 b_{0}} \stackrel{[0,1]}{\mathcal{P}^{\prime \prime}}{ }_{2 a_{0}} C_{c_{2}}^{a_{0} b_{0}} \bar{D}_{b_{2}}^{c_{2}} A_{a_{1}}^{b_{2}}\right)
\end{aligned}
$$

Formulae (87 88) help us to determine the concrete form of the redefinition under discussion

$$
\left(\stackrel{[1,0]}{\mathcal{P}}_{1 a_{0}}, \stackrel{[1,0]}{\mathcal{P}}_{1 a_{2}}, \stackrel{[0,1]}{\mathcal{P}}_{2 a_{0}}, \stackrel{[0,1]}{\mathcal{P}}_{2 a_{2}}\right) \rightarrow\left(\stackrel{[1,0]}{\mathcal{P}}_{1 a_{0}}, \stackrel{[1,0]}{\mathcal{P}}_{1 a_{2}},{ }^{[0,1]}{ }_{2 a_{0}}, \stackrel{[0,1]}{\mathcal{P}}_{2 a_{2}}\right)
$$

where

$$
\begin{gathered}
\stackrel{[1,0]}{\mathcal{P}}^{\prime \prime}{ }_{1 a_{0}}=R_{a_{0}}^{b_{0}} \stackrel{[1,0]}{\mathcal{P}}_{1 b_{0}}+Q_{a_{0}}^{b_{2}} \stackrel{[1,0]}{\mathcal{P}}_{1 b_{2}}, \\
\stackrel{[0,1]}{\mathcal{P}}_{2 a_{0}}=R_{a_{0}}^{b_{0}} \stackrel{[0,1]}{\mathcal{P}}{ }_{2 b_{0}}+Q_{a_{0}}^{b_{2}} \stackrel{[0,1]}{\mathcal{P}}_{2 b_{2}}, \\
R_{a_{0}}^{b_{0}}=\delta_{a_{0}}^{b_{0}}-Z_{b_{1}}^{b_{0}} A_{a_{0}}^{b_{1}}+C_{c_{2}}^{b_{0} c_{0}} G_{c_{0}} \bar{D}_{b_{2}}^{c_{2}} A_{a_{1}}^{b_{2}} A_{a_{0}}^{a_{1}}, Q^{b_{2}}{ }_{a_{0}}=-\bar{D}_{a_{2}}^{b_{2}} A_{a_{1}}^{a_{2}} A_{a_{0}}^{a_{1}} .
\end{gathered}
$$

As no nontrivial co-cycles connected with the initial reducibility appear at total resolution degree one, it follows that there will also be no nontrivial 
co-cycles correlated to this type of reducibility at higher resolution degrees. Consequently, the constraints underlying the new Koszul-Tate bicomplex

$$
\gamma_{a_{0}} \equiv G_{a_{0}}+A_{a_{0}}^{a_{1}} \pi_{a_{1}} \approx 0, \gamma_{a_{2}} \equiv Z_{a_{2}}^{a_{1}} \pi_{a_{1}} \approx 0
$$

are truly irreducible. Moreover, they are also first-class as (96) leads, after some computation, to

$$
\begin{aligned}
& \pi_{a_{1}}=\gamma_{a_{0}}\left(Z_{a_{1}}^{a_{0}}-C_{c_{2}}^{a_{0} b_{0}} G_{b_{0}} \bar{D}_{b_{2}}^{c_{2}} A_{a_{1}}^{b_{2}}\right)+\gamma_{b_{2}} \bar{D}_{a_{2}}^{b_{2}} A_{a_{1}}^{a_{2}} \\
& G_{a_{0}}=\gamma_{b_{0}}\left(\delta_{a_{0}}^{b_{0}}-Z_{b_{1}}^{b_{0}} A_{a_{0}}^{b_{1}}+C_{c_{2}}^{b_{0} c_{0}} G_{c_{0}} \bar{D}_{b_{2}}^{c_{2}} A_{b_{1}}^{b_{2}} A_{a_{0}}^{b_{1}}\right)- \\
& \gamma_{b_{2}} \bar{D}_{a_{2}}^{b_{2}} A_{a_{1}}^{a_{2}} A_{a_{0}}^{a_{1}} .
\end{aligned}
$$

Evaluating the Poisson brackets among the constraint functions $\left(\gamma_{a_{0}}, \gamma_{a_{2}}\right)$ with the help of (97 98), we find that they vanish weakly on the surface $\gamma_{a_{0}} \approx 0, \gamma_{a_{2}} \approx 0$, hence the constraints (96) are first-class.

Then, we can conclude that in the case $L=2$ we succeeded again in switching from an original reducible Koszul-Tate bicomplex associated with the second-stage reducible first-class constraints (1) to an irreducible one, based on the generators

$$
\left.\left(\stackrel{[0,0]^{A}}{z}, \underset{[0,0]}{z}\right]^{A_{1}}\right),\left(\stackrel{[1,0]}{\mathcal{P}}_{1 a_{0}}, \stackrel{[1,0]}{\mathcal{P}}_{1 a_{2}}, \stackrel{[0,1]}{\mathcal{P}}_{2 a_{0}}, \stackrel{[0,1]}{\mathcal{P}}_{2 a_{2}}\right),\left(\begin{array}{cc}
{[1,1]} & {[1,1]} \\
\lambda_{a_{0}}, & \lambda_{a_{2}}
\end{array}\right),
$$

and defined by the relations $(67-70)$ and $(7274)$, where the irreducible firstclass constraint functions $\gamma_{a_{0}}, \gamma_{a_{2}}$ are given in (96). It can be verified directly that the above-mentioned relations define a correct irreducible Koszul-Tate bicomplex, which agrees with the basic conditions (15 17), with $\delta_{R a}$ and $C^{\infty}(\Sigma)$ replaced by $\delta_{a}$, respectively, $C^{\infty}\left(\Sigma^{\prime \prime}\right)$, where $\Sigma^{\prime \prime}: \gamma_{a_{0}} \approx 0, \gamma_{a_{2}} \approx$ 0 . We cannot stress enough that it is precisely the requirement on the $\delta_{a^{-}}$ exactness of the new momenta $\pi_{a_{1}}$ which allows us to deduce an appropriate transformation of the antighosts at total resolution degree one (see (92 95)) that enforces the irreducibility.

\subsection{Generalization: $L$-stage reducible theories}

Now, after having analysed in detail the construction of an irreducible Koszul-

Tate bicomplex starting with an original first- or second-stage reducible set 
of first-class constraints, we are able to generalize our irreducible treatment to some initial on-shell $L$-stage reducible first-class constraints, described by the relations (15). Going along the same line as before, we enlarge the phase-space with the bosonic canonical pairs $\stackrel{[0,0]^{A_{2 k+1}}}{z}=\left(y^{a_{2 k+1}}, \pi_{a_{2 k+1}}\right)_{k=0, \cdots, \Lambda}$ and construct an irreducible Koszul-Tate bicomplex based on the generators

$$
\left.(\underset{[0,0]}{z}]^{A},\left(\underset{[0,0]^{A_{2 k+1}}}{z}\right)_{k=0, \cdots, \Lambda}\right),\left(\stackrel{[1,0]}{\mathcal{P}}_{1 a_{2 k},} \stackrel{[0,1]}{\mathcal{P}}_{2 a_{2 k},} \stackrel{[1,1]}{\lambda}_{a_{2 k}}\right)_{k=0, \cdots, \Gamma},
$$

and defined by the relations

$$
\begin{gathered}
\delta_{a} z^{A}=0, \delta_{a} z^{A_{2 k+1}}=0, k=0, \cdots, \Lambda, a=1,2, \\
\delta_{1} \stackrel{[1,0]}{\mathcal{P}}_{1 a_{2 k}}=-\gamma_{a_{2 k}}, \delta_{2} \stackrel{[1,0]}{\mathcal{P}}_{1 a_{2 k}}=0, k=0, \cdots, \Gamma, \\
\delta_{1} \stackrel{[0,1]}{\mathcal{P}}_{2 a_{2 k}}=0, \delta_{2} \stackrel{[0,1]}{\mathcal{P}}_{2 a_{2 k}}=-\gamma_{a_{2 k}}, k=0, \cdots, \Gamma, \\
\delta_{1} \stackrel{[1,1]}{\lambda}_{a_{2 k}}=-\stackrel{[0,1]}{\mathcal{P}}_{2 a_{2 k}}, \delta_{2} \stackrel{[1,1]}{\lambda}_{a_{2 k}}=\stackrel{[1,0]}{\mathcal{P}}_{1 a_{2 k}}, k=0, \cdots, \Gamma,
\end{gathered}
$$

which shows the irreducible constraints

$$
\begin{aligned}
& \gamma_{a_{0}} \equiv G_{a_{0}}+A_{a_{0}}^{a_{1}} \pi_{a_{1}} \approx 0 \\
& \gamma_{a_{2 k}} \equiv Z_{a_{2 k}}^{a_{2 k-1}} \pi_{a_{2 k-1}}+A_{a_{2 k}}^{a_{2 k+1}} \pi_{a_{2 k+1}} \approx 0, k=1, \cdots, \Gamma .
\end{aligned}
$$

The irreducibility is guaranteed by the presence of the functions $A_{a_{k}}^{a_{k+1}}$ in (105 106), which may involve at most the original variables $z^{A}$ and are chosen to satisfy the conditions

$$
\begin{gathered}
\operatorname{rank}\left(D_{b_{k}}^{a_{k}}\right) \approx \sum_{i=k}^{L}(-)^{k+i} M_{i}, k=1, \cdots, L-1, \\
\operatorname{rank}\left(D_{b_{L}}^{a_{L}}\right)=M_{L},
\end{gathered}
$$

where $D_{b_{k}}^{a_{k}}=Z_{b_{k}}^{a_{k-1}} A_{a_{k-1}}^{a_{k}}$. Acting like in the cases $L=1,2$, after some computation we find the relations

$$
\pi_{a_{2 k+1}}=\nu_{a_{2 k+1}}^{a_{2 j}} \gamma_{a_{2 j}}, k=0, \cdots, \Lambda
$$




$$
G_{a_{0}}=\nu_{a_{0}}^{a_{2 j}} \gamma_{a_{2 j}}
$$

for some functions $\nu_{a_{2 k+1}}^{a_{2 j}}$ and $\nu_{a_{0}}^{a_{2 j}}$. Computing the Poisson brackets among the constraint functions in (105 106), we find that they vanish weakly on the surface (105 106), hence they form a first-class set. The antighosts of the type $\stackrel{[1,0]}{\mathcal{P}}_{1 a_{2 k}}$ or $\stackrel{[0,1]}{\mathcal{P}}_{2 a_{2 k}}$ are fermionic, those denoted by $\stackrel{[1,1]}{\lambda}_{a_{2 k}}$ are bosonic, while the notations $\Lambda$ and $\Gamma$ signify

$$
\Lambda=\left\{\begin{array}{c}
\frac{L-1}{2}, \text { for } L \text { odd }, \\
\frac{L}{2}-1, \text { for } L \text { even, }
\end{array} \quad \Gamma=\left\{\begin{array}{l}
\frac{L-1}{2}, \text { for } L \text { odd } \\
\frac{L}{2}, \text { for } L \text { even }
\end{array}\right.\right.
$$

In order to avoid confusion, we use the conventions $f^{a_{k}}=0$ if $k<0$ or $k>L$.

With these elements at hand, it is obviously that the Koszul-Tate operators satisfy the fundamental requirements (15 17), with $\delta_{R a}$ and $C^{\infty}(\Sigma)$ replaced by $\delta_{a}$, respectively, $C^{\infty}(\bar{\Sigma})$, where

$$
\bar{\Sigma}: \gamma_{a_{2 k}} \approx 0, k=0, \cdots, \Gamma \text {. }
$$

This ends the general construction of an irreducible Koszul-Tate bicomplex associated with the original on-shell $L$-stage reducible Hamiltonian system.

\section{Irreducible Hamiltonian BRST-anti-BRST symmetry}

\subsection{Construction of the irreducible Hamiltonian BRST- anti-BRST symmetry}

Once we have accomplished the construction of the Koszul-Tate bicomplex based on the irreducible first-class constraints (105 106), it is natural to derive the irreducible Hamiltonian BRST-anti-BRST symmetry associated

with this constraint set. The BRST and anti-BRST operators $\left(s_{a}\right)_{a=1,2}$ corresponding to this irreducible first-class set should anticommute

$$
s_{a} s_{b}+s_{b} s_{a}=0, a, b=1,2,
$$

each of the two differentials splitting as

$$
s_{a}=\delta_{a}+D_{a}+\cdots, a=1,2,
$$


where $\left(\delta_{a}\right)_{a=1,2}$ generate the irreducible Koszul-Tate bicomplex, $\left(D_{a}\right)_{a=1,2}$ define the irreducible exterior longitudinal bicomplex, and the other terms (if necessary), generically denoted by "...", implement the anticommutation relations (113). The construction of the irreducible Koszul-Tate bicomplex has been elucidated in the previous subsections. With regard to the irreducible exterior longitudinal bicomplex, we remark that its generators are given by

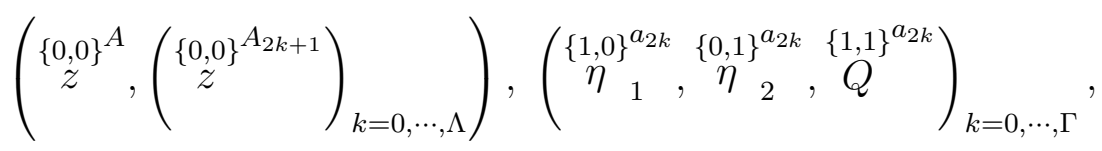

where $\stackrel{\{1,0\}}{\eta}_{1}^{a_{2 k}}, \stackrel{\{0,1\}}{\eta}_{2}^{a_{2 k}}$ are fermionic, $\stackrel{\{1,1\}^{a_{2 k}}}{Q}$ are bosonic, and $\stackrel{\{m, n\}}{F}$ represents an element of form bidegree biform $(F)=(m, n)$. For notational simplicity, we redenote the first-class constraints (105 106) by $\gamma_{\bar{\Delta}} \equiv\left(\gamma_{a_{2 k}}\right)_{k=0, \cdots, \Gamma} \approx$ 0 , such that we can equivalently rewrite the ghost spectrum in (115) like $\{1,0\}^{\bar{\Delta}}\{0,1\}^{\bar{\Delta}}\{1,1\}^{\bar{\Delta}}$

$\eta_{1}, \stackrel{\eta}{\eta}_{2}, \stackrel{Q}{Q}$. Due on the one hand to the first-class character of the constraints 105 106)

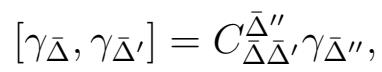

where $C_{\bar{\Delta}}^{\bar{\Delta}_{\bar{\Delta}^{\prime}}^{\prime \prime}}$ denote the first-order structure functions, and on the other hand, to their irreducibility, it follows that the actions of $\left(D_{a}\right)_{a=1,2}$ on the generators from the exterior longitudinal bicomplex are completely defined through the relations

$$
\begin{aligned}
& D_{1} \stackrel{\{0,0\}}{F}=\left[\stackrel{\{0,0\}}{F}, \gamma_{\bar{\Delta}}\right] \stackrel{\{1,0\}}{\eta}_{1}^{\bar{\Delta}}, D_{2} \stackrel{\{0,0\}}{F}=\left[\stackrel{\{0,0\}}{F}, \gamma_{\bar{\Delta}}\right] \stackrel{\{0,1\}}{\eta}_{2}^{\bar{\Delta}}, \\
& D_{1} \stackrel{\{1,0\}}{\eta}_{1}^{\bar{\Delta}}=\frac{1}{2} C_{\overline{\bar{\Delta}}^{\prime} \bar{\Delta}^{\prime \prime}}^{\overline{\bar{\Delta}}} \stackrel{\{1,0\}}{\eta}_{1}^{\bar{\Delta}^{\prime}} \stackrel{\{1,0\}}{\eta}_{1}^{\bar{\Delta}^{\prime \prime}} \\
& D_{2} \stackrel{\{1,0\}}{\eta}_{1}^{\bar{\Delta}}=\stackrel{\{1,1\}}{Q}^{\bar{\Delta}}+\frac{1}{2} C_{\bar{\Delta}^{\prime} \bar{\Delta}^{\prime \prime}}^{\overline{\bar{s}}^{\prime}} \stackrel{\{1,0\}}{\eta}_{1}^{\bar{\Delta}^{\prime}}\left\{\stackrel{\{0,1\}}{\eta}_{2}^{\bar{\Delta}^{\prime \prime}}\right. \\
& D_{1} \stackrel{\{0,1\}}{\eta}_{2}^{\bar{\Delta}}=-\stackrel{\{1,1\}}{Q}^{\bar{\Delta}}+\frac{1}{2} C_{\bar{\Delta}^{\prime} \bar{\Delta}^{\prime \prime}} \stackrel{\{0,1\}}{\eta}_{2}^{\bar{\Delta}^{\prime}}\{1,0\}_{1}^{\bar{\Delta}^{\prime \prime}} \\
& D_{2} \stackrel{\{0,1\}}{\eta}_{2}^{\bar{\Delta}}=\frac{1}{2} C_{\bar{\Delta}^{\prime} \bar{\Delta}^{\prime \prime}} \stackrel{\{0,1\}}{\eta}_{2}^{\bar{\Delta}^{\prime}}\left\{\stackrel{\{0,1\}}{\eta}_{2}^{\bar{\Delta}^{\prime \prime}},\right.
\end{aligned}
$$




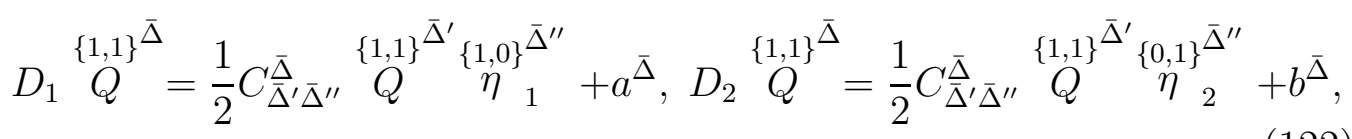

where $\stackrel{\{0,0\}}{F}$ can be any function of the variables $z^{A},\left(z^{A_{2 k+1}}\right)_{k=0, \cdots, \Lambda}$, and the functions $a^{\bar{\Delta}}$ and $b^{\bar{\Delta}}$ read as $a^{\bar{\Delta}}=\frac{1}{8} C C_{\bar{\Delta}_{1} \bar{\Delta}^{\prime}} C_{\overline{\bar{\Delta}}_{2} \bar{\Delta}_{3}}^{\bar{\Delta}_{3}} \stackrel{\{0,1\}}{\eta}_{2}^{\bar{\Delta}_{1}} \stackrel{\{1,0\}}{\eta}_{1}^{\bar{\Delta}_{2}} \stackrel{\{1,0\}}{\eta}_{1}^{\bar{\Delta}_{3}}$, respec-

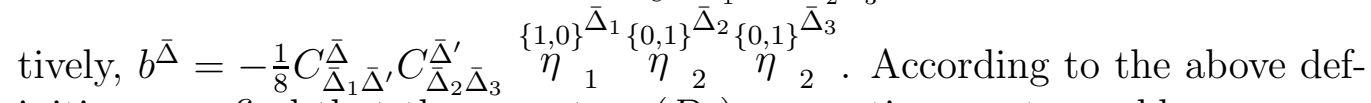
initions, we find that the operators $\left(D_{a}\right)_{a=1,2}$ anticommute weakly

$$
D_{a} D_{b}+D_{b} D_{a} \approx 0, a, b=1,2,
$$

where the weak equality is referring to the first-class surface (105 106). Moreover, we infer that the cohomology spaces of $\left(D_{a}\right)_{a=1,2}$ at form bidegree $(0,0)$ are given by the algebra of physical observables corresponding to the irreducible first-class constraints (105 106). Then, we infer that the exterior longitudinal bicomplex associated with the irreducible system meets all the requirements of the Hamiltonian BRST-anti-BRST method.

If we extend the actions of $\left(\delta_{a}\right)_{a=1,2}$ to the ghosts through

$$
\delta_{a} \eta_{1}^{\bar{\Delta}}=0, \delta_{a} \eta_{2}^{\bar{\Delta}}=0, \delta_{a} Q^{\bar{\Delta}}=0
$$

and set bires $\left(\eta_{1}^{\bar{\Delta}}\right)=(0,0)$, bires $\left(\eta_{2}^{\bar{\Delta}}\right)=(0,0)$, bires $\left(Q^{\bar{\Delta}}\right)=(0,0)$, we rediscover the relations

$$
\begin{gathered}
\delta_{a} \delta_{b}+\delta_{b} \delta_{a}=0, a, b=1,2, \\
H_{0,0}\left(\delta_{a}\right)=C^{\infty}(\bar{\Sigma}), a=1,2, \\
H_{j, k}\left(\delta_{a}\right)=0, j, k \geq 0, j+k \neq 0, a=1,2,
\end{gathered}
$$

which confirms that the irreducible Koszul-Tate differentials can be properly prolonged to the entire BRST-anti-BRST generator algebra, i.e. such that to preserve the biresolution property. Furthermore, the first-class character of the irreducible constraints (105 106) ensures that the irreducible exterior longitudinal derivatives $\left(D_{a}\right)_{a=1,2}$ (and their accompanying bigrading, biform) can also be extended to the antighosts in such a way as to become differentials modulo $\left(\delta_{a}\right)_{a=1,2}$. Consequently, we can consistently construct 
[5] the Hamiltonian BRST-anti-BRST symmetry of the irreducible theory, realized via two anticommuting differentials $\left(s_{a}\right)_{a=1,2}$

$$
s_{a} s_{b}+s_{b} s_{a}=0, a, b=1,2,
$$

that start like

$$
s_{a}=\delta_{a}+D_{a}+\cdots, a=1,2,
$$

and are bigraded accordingly with the new ghost bidegree bingh $=\left(n g h_{1}, n g h_{2}\right)$, defined like in (8). The generators of the superalgebra underlying the irreducible Hamiltonian BRST-anti-BRST bicomplex are precisely (100) and the ghosts in (115), but bigraded in terms of bingh. Then, the cohomology groups of $\left(s_{a}\right)_{a=1,2}$ at new ghost bidegree zero, $H^{0,0}\left(s_{a}\right)$, will be isomorphic to the algebra of physical observables corresponding to the irreducible system. In the following we will denote by $\stackrel{(m, n)}{F}$ an object with $\operatorname{bingh}(F)=(m, n)$.

\subsection{Relation with the reducible Hamiltonian BRST- anti-BRST symmetry}

Let us investigate now the relation between the Hamiltonian BRST-antiBRST symmetries of the reducible and irreducible systems. In view of this, we show that the physical observables of the two theories coincide. Let $F$ be an observable of the irreducible system. Consequently, it satisfies the equations

$$
\left[F, \gamma_{a_{2 k}}\right] \approx 0, k=0, \cdots, \Gamma,
$$

where the weak equality refers to the surface $\bar{\Sigma}$, given by (112). Using the relations (109 110), we then find that $F$ also fulfils the equations

$$
\begin{gathered}
{\left[F, G_{a_{0}}\right]=\left[F, \nu_{a_{0}}^{a_{2 j}}\right] \gamma_{a_{2 j}}+\left[F, \gamma_{a_{2 j}}\right] \nu_{a_{0}}^{a_{2 j}} \approx 0,} \\
{\left[F, \pi_{a_{2 k+1}}\right]=\left[F, \nu_{a_{2 k+1}}^{a_{2 j}}\right] \gamma_{a_{2 j}}+\left[F, \gamma_{a_{2 j}}\right] \nu_{a_{2 k+1}}^{a_{2 j}} \approx 0, k=0, \cdots, \Lambda,}
\end{gathered}
$$

on this surface. So, every observable of the irreducible theory should verify the equations (131-132) on $\bar{\Sigma}$. Now, we observe that this surface is equivalent to that described by the equations

$$
G_{a_{0}} \approx 0, \pi_{a_{2 k+1}} \approx 0, k=0, \cdots, \Lambda
$$


Indeed, it is clear that if (133) takes place, then (105 106) hold. The converse results from (109 110), which show that if (105 106) hold, then (133) are also valid. This proves the equivalence between the first-class surfaces $\bar{\Sigma}$ and (133). Consequently, we have that every observable of the irreducible theory, which we found that verifies equations (131 132) on $\bar{\Sigma}$, will check these equations also on the surface (133). This means that every observable of the irreducible system is an observable of the theory based on the firstclass constraints (133). Conversely, if $F$ represents a physical observable of the system subject to the constraints (133), then it should check the equations

$$
\left[F, G_{a_{0}}\right] \approx 0,\left[F, \pi_{a_{2 k+1}}\right] \approx 0, k=0, \cdots, \Lambda,
$$

on the surface (133). Then, on behalf of (105 106) it follows that $F$ satisfies the relations

$$
\begin{aligned}
& {\left[F, \gamma_{a_{0}}\right]=\left[F, G_{a_{0}}\right]+\left[F, A_{a_{0}}^{a_{1}}\right] \pi_{a_{1}}+\left[F, \pi_{a_{1}}\right] A_{a_{0}}^{a_{1}} \approx 0,} \\
& {\left[F, \gamma_{a_{2 k}}\right]=\left[F, Z_{a_{2 k}}^{a_{2 k-1}}\right] \pi_{a_{2 k-1}}+\left[F, \pi_{a_{2 k-1}}\right] Z_{a_{2 k}}^{a_{2 k-1}}+} \\
& {\left[F, A_{a_{2 k}}^{a_{2 k+1}}\right] \pi_{a_{2 k+1}}+\left[F, \pi_{a_{2 k+1}}\right] A_{a_{2 k}}^{a_{2 k+1}} \approx 0, k=1, \cdots, \Gamma,}
\end{aligned}
$$

on the same surface. Recalling once again the equivalence between this surface and $\bar{\Sigma}$, we find that $F$ will verify the equations (135 136) also on $\bar{\Sigma}$, and is therefore an observable of the irreducible system. From the above discussion we conclude that the physical observables of the irreducible theory coincide with those associated with the system subject to the first-class constraints (133). Next, we show that the physical observables of the system possessing the constraints (133) and those corresponding to the original reducible theory coincide. In this light, we remark that the surface (133) can be inferred in a trivial manner from (11) by adding the canonical pairs $\left(y^{a_{2 k+1}}, \pi_{a_{2 k+1}}\right)_{k=0, \cdots, \Lambda}$ and requiring that their momenta vanish. Thus, the observables of the original redundant theory are unaffected by the introduction of the new canonical pairs. In fact, the difference between an observable $F$ of the system subject to the constraints (133) and one of the original theory, $\bar{F}$, is of the type $F-\bar{F}=\sum_{k=0}^{\Lambda} f^{a_{2 k+1}} \pi_{a_{2 k+1}}$. As any two observables that differ through a combination of first-class constraint functions can be identified, we find that the physical observables of the initial theory coincide with 
those of the system described by the constraints (133). So far, we proved that the observables of the system with the constraints (133) coincide on the one hand with those of the irreducible theory, and, on the other hand, with those of the original reducible one. In conclusion, the physical observables associated with the irreducible system also coincide with those of the starting on-shell reducible first-class theory. In turn, this result will have a strong impact at the level of the BRST-anti-BRST analysis.

In the above we have shown that starting with an arbitrary on-shell reducible first-class Hamiltonian system displaying the Hamiltonian BRSTanti-BRST symmetry $\left(s_{R a}\right)_{a=1,2}$ we can construct a corresponding irreducible first-class theory, whose BRST-anti-BRST symmetry $\left(s_{a}\right)_{a=1,2}$ complies with the basic requirements of the Hamiltonian BRST-anti-BRST formalism. The previous result on the physical observables induces that the zeroth order cohomological groups of the reducible and irreducible BRST and anti-BRST operators are isomorphic

$$
H^{0,0}\left(s_{R a}\right) \simeq H^{0,0}\left(s_{b}\right), a, b=1,2 .
$$

In addition, each symmetry is generated by a couple of anticommuting differentials

$$
s_{R a} s_{R b}+s_{R b} s_{R a}=0=s_{a} s_{b}+s_{b} s_{a}, a, b=1,2 .
$$

Then, from the point of view of the fundamental equations of the BRSTanti-BRST formalism, namely, the nilpotency and anticommutativity of the BRST and anti-BRST operators, respectively, the isomorphism between the zeroth order cohomological groups of the BRST and anti-BRST differentials and the algebra of physical observables, it follows that it is permissible to replace the Hamiltonian BRST-anti-BRST symmetry of the original $L$-stage reducible system with that of the irreducible theory. Thus, we can substitute the path integral of the reducible system in the Hamiltonian BRST-antiBRST approach by that of the irreducible theory.

However, it would be convenient to infer a covariant path integral with respect to the irreducible system. The present phase-space coordinates may not ensure the covariance. For instance, if we analyse the gauge transformations of the extended action of the irreducible system, we remark that those corresponding to the Lagrange multipliers of the constraint functions $\gamma_{a_{0}}$ will not involve the term $-Z_{a_{1}}^{a_{0}} \epsilon^{a_{1}}$, which is present within the reducible 
context with respect to the constraint functions $G_{a_{0}}$. For all known models, the presence of this term is essential in arriving at some covariant gauge transformations at the Lagrangian level. For this reason it is necessary to gain such a term also within the irreducible setting. Moreover, it is possible that some of the newly added canonical variables lack covariant Lagrangian gauge transformations. This signalizes that we need to add more phase-space variables to be constrained in an appropriate manner. In view of this, we introduce the additional bosonic canonical pairs

$$
\begin{gathered}
\left(y^{(1) a_{2 k+1}}, \pi_{a_{2 k+1}}^{(1)}\right),\left(y^{(2) a_{2 k+1}}, \pi_{a_{2 k+1}}^{(2)}\right), k=0, \cdots, \Lambda, \\
\left(y^{a_{2 k}}, \pi_{a_{2 k}}\right), k=1, \cdots, \Gamma,
\end{gathered}
$$

subject to some constraints of the type

$$
\begin{gathered}
\gamma_{a_{2 k+1}}^{(1)} \equiv \pi_{a_{2 k+1}}-\pi_{a_{2 k+1}}^{(1)} \approx 0, \gamma_{a_{2 k+1}}^{(2)} \equiv \pi_{a_{2 k+1}}^{(2)} \approx 0, k=0, \cdots, \Lambda, \\
\gamma_{a_{2 k}}^{(1)} \equiv \pi_{a_{2 k}} \approx 0, k=1, \cdots, \Gamma .
\end{gathered}
$$

In this manner we do not affect in any way the properties of the irreducible theory as (141 142) still form together with (105 106) an irreducible first-class set. The newly added constraints implies the introduction of some supplementary ghosts and antighosts and the extension of the action of the BRST and anti-BRST operators on them in the usual manner. Then, there exists a consistent Hamiltonian BRST-anti-BRST symmetry with respect to the new irreducible theory, described by the constraints (105 106) and (141 142). Now, if we choose the first-class Hamiltonian with respect to the above first-class constraints in an adequate manner, we can, in principle, generate a gauge algebra that leads to some covariant Lagrangian gauge transformations. ¿From (109) it results that the former set of constraints in (141) reduces to $\pi_{a_{2 k+1}}^{(1)} \approx 0$. Thus, we observe that the surface (105 106), (141 142) results in a trivial way from (105 106) by adding the canonical variables (139 140), and demanding that their momenta vanish. Then, the difference between an observable $F$ of the new irreducible theory and one of the previous irreducible system, $\bar{F}$, is of the type $F-\bar{F}=\sum_{k=0}^{\Lambda} f^{a_{2 k+1}} \pi_{a_{2 k+1}}^{(1)}+\sum_{k=0}^{\Lambda} g^{a_{2 k+1}} \pi_{a_{2 k+1}}^{(2)}+\sum_{k=1}^{\Gamma} h^{a_{2 k}} \pi_{a_{2 k}}$, hence $F$ and $\bar{F}$ can be identified. Therefore, the physical observables corresponding to the two irreducible systems coincide, such that the supplementary constraints (141-142) do not afflict the previously established equivalence with 
the physical observables of the original redundant theory. Consequently, we can replace the Hamiltonian BRST-anti-BRST symmetry of the original reducible system with that of the latter irreducible theory, and similarly with regard to the associated path integrals.

¿From now on, the Hamiltonian BRST-anti-BRST quantization of the irreducible theory follows the standard lines. Defining a canonical action for $\left(s_{a}\right)_{a=1,2}$ in the usual way as $s_{a} \bullet=\left[\bullet, \Omega_{a}\right]$, with $\left(\Omega_{a}\right)_{a=1,2}$ the BRST, respectively, anti-BRST charge, the nilpotency and anticommutativity of $s_{a}$ imply that $\Omega_{a}$ should satisfy the equations

$$
\left[\Omega_{a}, \Omega_{b}\right]=0, a, b=1,2,
$$

where $\operatorname{bingh}\left(\Omega_{1}\right)=(1,0)$, bingh $\left(\Omega_{2}\right)=(0,1)$. The existence of the solution to equations (143) is then guaranteed by the biacyclicity of the irreducible Koszul-Tate bicomplex at positive total resolution degrees. Once we have constructed the total BRST-anti-BRST charge, $\Omega^{T}=\Omega_{1}+\Omega_{2}$, we pass to the construction of the BRST-anti-BRST-invariant Hamiltonian, $H_{B}^{T}=H^{\prime}+$ "more", that satisfies bingh $\left(H_{B}^{T}\right)=(0,0),\left[H_{B}^{T}, \Omega^{T}\right]=0$, where $H^{\prime}$ denotes the first-class Hamiltonian with respect to the constraints (105106) and (141 142). In order to fix the gauge, we have to choose a gaugefixing fermion $K^{T}$ that implements some irreducible gauge conditions and which is taken such that $\left(s_{1}+s_{2}\right) K^{T}=\left[K^{T}, \Omega^{T}\right]$ contains only terms of new ghost bigrading of the form $(k, k)$. It has been shown in 5 that under these circumstances the gauge-fixed Hamiltonian $H_{K}^{T}=H_{B}^{T}+\left[K^{T}, \Omega^{T}\right]$ is both BRST and anti-BRST invariant and, moreover, produces a correct gauge-fixed action $S_{K}^{T}$ with respect to the irreducible theory. In this way, we showed how a reducible first-class Hamiltonian system can be approached along an irreducible BRST-anti-BRST procedure. This completes our treatment.

\section{Example: the Freedman-Townsend model}

The starting point is the Lagrangian action of the Freedman-Townsend model [31]

$$
S_{0}^{L}\left[A_{\mu}^{a}, B_{a}^{\mu \nu}\right]=\frac{1}{2} \int d^{4} x\left(-B_{a}^{\mu \nu} F_{\mu \nu}^{a}+A_{\mu}^{a} A_{a}^{\mu}\right)
$$


where $B_{a}^{\mu \nu}$ denotes a set of antisymmetric tensor fields, and the field strength of $A_{\mu}^{a}$ reads as $F_{\mu \nu}^{a}=\partial_{\mu} A_{\nu}^{a}-\partial_{\nu} A_{\mu}^{a}-f_{b c}^{a} A_{\mu}^{b} A_{\nu}^{c}$. The canonical analysis of this theory outputs the constraints

$$
\begin{gathered}
\Phi_{i}^{(1) a} \equiv \epsilon_{0 i j k} \pi^{j k a} \approx 0, \Phi_{i}^{(2) a} \equiv \frac{1}{2} \epsilon_{0 i j k}\left(F^{j k a}-\left(D^{[j}\right)^{a}{ }_{b} \pi^{k] 0 b}\right) \approx 0 \\
\chi_{i}^{(1) a} \equiv \pi_{0 i}^{a} \approx 0, \chi_{i}^{(2) a} \equiv \pi_{i}^{a}+B_{0 i}^{a} \approx 0, \chi_{a}^{(1)} \equiv \pi_{a}^{0} \approx 0 \\
\chi_{a}^{(2)} \equiv A_{a}^{0}+f_{a b}^{c} B_{c}^{0 i} \pi_{0 i}^{b}+\left(D_{i}\right)_{a}{ }^{b} \pi_{b}^{i} \approx 0
\end{gathered}
$$

and the first-class Hamiltonian

$$
\begin{aligned}
& H=\int d^{3} x\left(\frac{1}{2} B_{a}^{i j}\left(F_{i j}^{a}-\left(D_{[i}\right)^{a}{ }_{b} \pi_{j] 0}^{b}\right)-\frac{1}{2} A_{\mu}^{a} A_{a}^{\mu}-\right. \\
& \left.A_{0}^{a}\left(\left(D_{i}\right)_{a}{ }^{b} \pi_{b}^{i}+f^{c}{ }_{a b} B_{c}^{0 i} \pi_{0 i}^{b}\right)-A_{a}^{i}\left(\pi_{0 i}^{a}-\partial_{i} \pi_{0}^{a}\right)\right) .
\end{aligned}
$$

The symbol $[i j]$ appearing in (148) signifies the antisymmetry with respect to the indices between brackets. In the above, the notations $\pi_{a}^{\mu}$ and $\pi_{\mu \nu}^{a}$ denote the momenta respectively conjugated in the Poisson bracket to the fields $A_{\mu}^{a}$ and $B_{a}^{\mu \nu}$, while the covariant derivatives are defined by $\left(D_{i}\right)^{a}{ }_{b}=\delta^{a}{ }_{b} \partial_{i}+f_{b c}^{a} A_{i}^{c}$ and $\left(D_{i}\right)_{b}{ }^{a}=\delta_{b}{ }^{a} \partial_{i}-f_{b c}^{a} A_{i}^{c}$. By computing the Poisson brackets between the constraint functions (145 147) we find that (145) are first-class and (146147) second-class. In addition, the functions $\Phi_{i}^{(2) a}$ from (145) are on-shell first-stage reducible

$$
\left(\left(D^{i}\right)_{b}^{a}+f_{b c}^{a} \pi^{0 i c}\right) \Phi_{i}^{(2) b}=-\epsilon^{0 i j k} f_{b c}^{a} \chi_{i}^{(1) b}\left(D_{j}\right)_{d}^{c} \chi_{k}^{(1) d} \approx 0
$$

In order to deal with the Hamiltonian BRST-anti-BRST formalism, it is useful to eliminate the second-class constraints with the help of the Dirac bracket [30] built with respect to themselves. By passing to the Dirac bracket, the constraints (146 147) can be regarded as strong equalities with the help of which we can express $A_{0}^{a}, \pi_{a}^{0}, \pi_{i}^{a}$ and $\pi_{0 i}^{a}$ in terms of the remaining fields and momenta, such that the independent 'co-ordinates' of the reduced phasespace are $A_{i}^{a}, B_{a}^{0 i}, B_{a}^{i j}$ and $\pi_{i j}^{a}$. The non-vanishing Dirac brackets among the independent components are expressed by

$$
\left[B_{a}^{0 i}(x), A_{j}^{b}(y)\right]_{x^{0}=y^{0}}^{*}=\delta_{a}{ }^{b} \delta^{i}{ }_{j} \delta^{3}(\mathbf{x}-\mathbf{y}),
$$




$$
\left[B_{a}^{i j}(x), \pi_{k l}^{b}(y)\right]_{x^{0}=y^{0}}^{*}=\frac{1}{2} \delta_{a}{ }^{b} \delta^{[i}{ }_{k} \delta^{j]} \delta^{3}(\mathbf{x}-\mathbf{y}) .
$$

In terms of the independent fields, the first-class constraints and first-class Hamiltonian take the form

$$
\begin{gathered}
\gamma_{i}^{(1) a} \equiv \epsilon_{0 i j k} \pi^{j k a} \approx 0, G_{i}^{(2) a} \equiv \frac{1}{2} \epsilon_{0 i j k} F^{j k a} \approx 0 \\
\tilde{H}=\frac{1}{2} \int d^{3} x\left(B_{a}^{i j} F_{i j}^{a}-A_{i}^{a} A_{a}^{i}+\left(\left(D^{i}\right)^{a}{ }_{b} B_{0 i}^{b}\right)\left(D_{j}\right)_{a}{ }^{c} B_{c}^{0 j}\right) \equiv \int d^{3} x \tilde{h},
\end{gathered}
$$

while the reducibility relations

$$
\left(D^{i}\right)_{a}^{b} G_{i}^{(2) a}=0
$$

hold off-shell in this case. Moreover, the first-class constraints (152) remain Abelian in terms of the Dirac bracket. In the following we work with the theory based on the reducible first-class constraints (152) and on the firstclass Hamiltonian (153), in the context of the Dirac bracket defined by (150 151).

In conclusion, this is an example of first-stage reducible theory $(L=1)$, with $G_{a_{0}} \rightarrow G_{i}^{(2) a}$ and $Z_{a_{1}}^{a_{0}} \rightarrow\left(D^{i}\right)^{b}{ }_{a}$. Acting like in subsection 3.1, we add the bosonic scalar pairs $\left(\varphi_{b}, \pi^{b}\right)$ (that play the role of the variables $\left(y^{a_{1}}, \pi_{a_{1}}\right)$ ) and take the matrix $A_{a_{0}}^{a_{1}}$ of the form

$$
A_{a_{0}}^{a_{1}} \rightarrow-\left(D_{i}\right)^{a}{ }_{b},
$$

such that condition (53) is indeed satisfied. Consequently, from (53) and (155) we deduce the irreducible first-class constraints associated with $G_{i}^{(2) a} \approx$ 0 of the type

$$
\gamma_{a_{0}} \approx 0 \rightarrow \gamma_{i}^{(2) a} \equiv \frac{1}{2} \epsilon_{0 i j k} F^{j k a}-\left(D_{i}\right)^{a}{ }_{b} \pi^{b} \approx 0
$$

Now, it is easy to see that the constraint functions in (156) and $\gamma_{i}^{(1) a}$ from (152) are also first-class and irreducible. In addition, as we mentioned at the end of the subsection 4.2 (see relations (139) and (141)), we enlarge the phase-space with the supplementary bosonic scalar pairs

$$
\left(y^{(1) a_{1}}, \pi_{a_{1}}^{(1)}\right) \rightarrow\left(\varphi_{a}^{(1)}, \pi^{(1) a}\right),\left(y^{(2) a_{1}}, \pi_{a_{1}}^{(2)}\right) \rightarrow\left(\varphi_{a}^{(2)}, \pi^{(2) a}\right),
$$


subject to the constraints

$$
\gamma_{a_{1}}^{(1)} \approx 0 \rightarrow \gamma^{(1) a} \equiv \pi^{a}-\pi^{(1) a} \approx 0, \gamma_{a_{1}}^{(2)} \approx 0 \rightarrow \gamma^{(2) a} \equiv-\pi^{(2) a} \approx 0
$$

whose presence does not in any way harm the irreducible theory, and, moreover, is helpful at deriving a covariant form of the path integral corresponding to the irreducible model. So far, we derived an irreducible system for the Freedman-Townsend model, based on the irreducible first-class constraints

$$
\begin{gathered}
\gamma_{i}^{(1) a} \equiv \epsilon_{0 i j k} \pi^{j k a} \approx 0, \gamma_{i}^{(2) a} \equiv \frac{1}{2} \epsilon_{0 i j k} F^{j k a}-\left(D_{i}\right)^{a}{ }_{b} \pi^{b} \approx 0 \\
\gamma^{(1) a} \equiv \pi^{a}-\pi^{(1) a} \approx 0, \gamma^{(2) a} \equiv-\pi^{(2) a} \approx 0 .
\end{gathered}
$$

The first-class Hamiltonian with respect to the above constraints can be chosen of the type

$$
\begin{aligned}
& H^{\prime} \equiv \int d^{3} x h^{\prime}=\int d^{3} x\left(\frac{1}{2} B_{a}^{i j}\left(F_{i j}^{a}+\epsilon_{0 i j k}\left(D^{k}\right)_{b}^{a} \pi^{b}\right)-\right. \\
& \frac{1}{2} A_{i}^{a} A_{a}^{i}+\varphi_{a} \pi^{(2) a}-\varphi_{a}^{(2)}\left(D_{i}\right)_{b}^{a}\left(D^{i}\right)_{c}^{b} \pi^{c}+ \\
& \left.\frac{1}{2}\left(\left(D_{i}\right)_{a}{ }^{b} B_{b}^{0 i}-f_{a b}^{c}\left(\varphi_{c} \pi^{b}+\varphi_{c}^{(1)} \pi^{b}+\varphi_{c}^{(2)} \pi^{(2) b}\right)\right)^{2}\right)
\end{aligned}
$$

where we employed the notation

$$
\begin{aligned}
& \left(\left(D_{i}\right)_{a}{ }^{b} B_{b}^{0 i}-f_{a b}^{c}\left(\varphi_{c} \pi^{b}+\varphi_{c}^{(1)} \pi^{b}+\varphi_{c}^{(2)} \pi^{(2) b}\right)\right)^{2} \equiv \\
& \left(\left(D_{i}\right)_{a}^{b} B_{b}^{0 i}-f_{a b}^{c}\left(\varphi_{c} \pi^{b}+\varphi_{c}^{(1)} \pi^{b}+\varphi_{c}^{(2)} \pi^{(2) b}\right)\right) \times \\
& \left(\left(D^{j}\right)_{d}^{a} B_{0 j}^{d}-f_{d e}^{a}\left(\pi^{d} \varphi^{e}+\pi^{d} \varphi^{(1) e}+\pi^{(2) d} \varphi^{(2) e}\right)\right) .
\end{aligned}
$$

The irreducible first-class constraints are Abelian, while the remaining gauge algebra relations read as

$$
\begin{aligned}
& {\left[\gamma_{i}^{(1) a}, H^{\prime}\right]^{*}=-\gamma_{i}^{(2) a},\left[\gamma_{i}^{(2) a}, H^{\prime}\right]^{*}=-\left(D_{i}\right)_{b}^{a} \gamma^{(2) b}+} \\
& f_{b c}^{a}\left(\left(D^{j}\right)_{d}^{b} B_{0 j}^{d}-f^{b}{ }_{d e}\left(\pi^{d} \varphi^{e}+\pi^{d} \varphi^{(1) e}+\pi^{(2) d} \varphi^{(2) e}\right)\right) \gamma_{i}^{(2) c}, \\
& {\left[\gamma^{(1) a}, H^{\prime}\right]^{*}=\gamma^{(2) a},\left[\gamma^{(2) a}, H^{\prime}\right]^{*}=\left(D^{i}\right)^{a}{ }_{b} \gamma_{i}^{(2) b}+} \\
& f_{b c}^{a}\left(\left(D^{j}\right)_{d}^{b} B_{0 j}^{d}-f^{b}{ }_{d e}\left(\pi^{d} \varphi^{e}+\pi^{d} \varphi^{(1) e}+\pi^{(2) d} \varphi^{(2) e}\right)\right) \gamma^{(2) c}
\end{aligned}
$$


As will be emphasized, the gauge algebra (163 164) ensures the Lorentz covariance of the irreducible approach.

Next, we determine the path integral of the irreducible first-class theory described by (159 161), associated with the Freedman-Townsend model, in the context of the Hamiltonian BRST-anti-BRST formalism. In view of this, we introduce the generators of the BRST-anti-BRST bicomplex

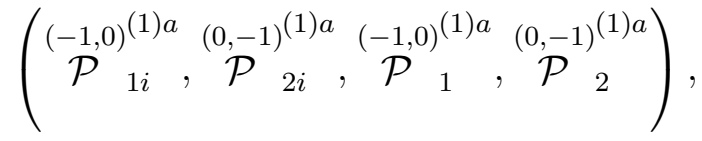

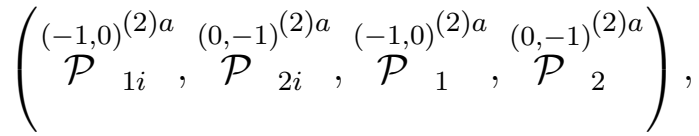

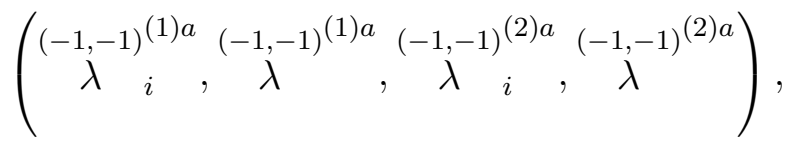

$$
\begin{aligned}
& \left(\stackrel{(1,0)}{\eta}_{1 a}^{(1) i}, \stackrel{(0,1)}{\eta}_{2 a}^{(1) i}, \stackrel{(1,0)}{\eta}_{1 a}^{(1)}, \stackrel{(0,1)}{\eta}_{2 a}^{(1)}\right)
\end{aligned}
$$

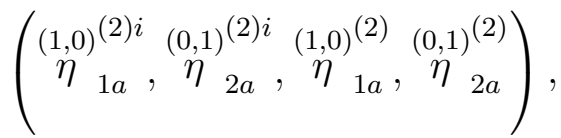

$$
\begin{aligned}
& \left(\begin{array}{cccc}
(1,1)^{(1) i} & (1,1)^{(1)} & (1,1)^{(2) i} & (1,1)^{(2)} \\
Q_{a} & , & Q_{a} \\
& &
\end{array}\right),
\end{aligned}
$$

graded according to the new ghost bidegree. The total BRST-anti-BRST charge has the form

$$
\begin{aligned}
& \Omega^{T}=\int d^{3} x\left(\gamma_{i}^{(1) a}\left(\eta_{1 a}^{(1) i}+\eta_{2 a}^{(1) i}\right)+\gamma_{i}^{(2) a}\left(\eta_{1 a}^{(2) i}+\eta_{2 a}^{(2) i}\right)+\gamma^{(1) a}\left(\eta_{1 a}^{(1)}+\eta_{2 a}^{(1)}\right)+\right. \\
& \gamma^{(2) a}\left(\eta_{1 a}^{(2)}+\eta_{2 a}^{(2)}\right)+Q_{a}^{(1) i}\left(\mathcal{P}_{1 i}^{(1) a}-\mathcal{P}_{2 i}^{(1) a}\right)+Q_{a}^{(2) i}\left(\mathcal{P}_{1 i}^{(2) a}-\mathcal{P}_{2 i}^{(2) a}\right)+ \\
& \left.Q_{a}^{(1)}\left(\mathcal{P}_{1}^{(1) a}-\mathcal{P}_{2}^{(1) a}\right)+Q_{a}^{(2)}\left(\mathcal{P}_{1}^{(2) a}-\mathcal{P}_{2}^{(2) a}\right)\right) .
\end{aligned}
$$

The BRST-anti-BRST-invariant Hamiltonian corresponding to the first-class Hamiltonian (161) is given by

$$
H_{B}^{T}=H^{\prime}+\int d^{3} x\left(\eta_{1 a}^{(1) i} \mathcal{P}_{1 i}^{(2) a}+\eta_{2 a}^{(1) i} \mathcal{P}_{2 i}^{(2) a}+Q_{a}^{(1) i} \lambda_{i}^{(2) a}-\eta_{1 a}^{(1)} \mathcal{P}_{1}^{(2) a}-\eta_{2 a}^{(1)} \mathcal{P}_{2}^{(2) a}-\right.
$$




$$
\begin{aligned}
& Q_{a}^{(1)} \lambda^{(2) a}+\eta_{1 a}^{(2) i}\left(D_{i}\right)^{a}{ }_{b} \mathcal{P}_{1}^{(2) b}+\eta_{2 a}^{(2) i}\left(D_{i}\right)^{a}{ }_{b} \mathcal{P}_{2}^{(2) b}+Q_{a}^{(2) i}\left(D_{i}\right)^{a}{ }_{b} \lambda^{(2) b}- \\
& \eta_{1 a}^{(2)}\left(D^{i}\right)^{a}{ }_{b} \mathcal{P}_{1 i}^{(2) b}-\eta_{2 a}^{(2)}\left(D^{i}\right)^{a}{ }_{b} \mathcal{P}_{2 i}^{(2) b}-Q_{a}^{(2)}\left(D^{i}\right)^{a}{ }_{b} \lambda_{i}^{(2) b}- \\
& f^{c}{ }_{a b}\left(\eta_{1 c}^{(2)} \mathcal{P}_{1}^{(2) b}+\eta_{2 c}^{(2)} \mathcal{P}_{2}^{(2) b}+Q_{c}^{(2)} \lambda^{(2) b}+\eta_{1 c}^{(2) i} \mathcal{P}_{1 i}^{(2) b}+\eta_{2 c}^{(2) i} \mathcal{P}_{2 i}^{(2) b}+Q_{c}^{(2) i} \lambda_{i}^{(2) b}\right) \times \\
& \times\left(\left(D^{j}\right)^{a}{ }_{d} B_{0 j}^{d}-f^{a}{ }_{d e}\left(\pi^{d} \varphi^{e}+\pi^{d} \varphi^{(1) e}+\pi^{(2) d} \varphi^{(2) e}\right)\right)+ \\
& \frac{1}{2}\left(f ^ { c } { } _ { a b } \left(\eta_{1 c}^{(2)} \mathcal{P}_{1}^{(2) b}+\eta_{2 c}^{(2)} \mathcal{P}_{2}^{(2) b}+Q_{c}^{(2)} \lambda^{(2) b}+\right.\right. \\
& \left.\left.\left.\eta_{1 c}^{(2) i} \mathcal{P}_{1 i}^{(2) b}+\eta_{2 c}^{(2) i} \mathcal{P}_{2 i}^{(2) b}+Q_{c}^{(2) i} \lambda_{i}^{(2) b}\right)\right)^{2}\right)
\end{aligned}
$$

where we use the notation

$$
\begin{aligned}
& \left(f ^ { c } { } _ { a b } \left(\eta_{1 c}^{(2)} \mathcal{P}_{1}^{(2) b}+\eta_{2 c}^{(2)} \mathcal{P}_{2}^{(2) b}+Q_{c}^{(2)} \lambda^{(2) b}+\right.\right. \\
& \left.\left.\eta_{1 c}^{(2) i} \mathcal{P}_{1 i}^{(2) b}+\eta_{2 c}^{(2) i} \mathcal{P}_{2 i}^{(2) b}+Q_{c}^{(2) i} \lambda_{i}^{(2) b}\right)\right)^{2} \equiv \\
& -f^{c}{ }_{a b}\left(\eta_{1 c}^{(2)} \mathcal{P}_{1}^{(2) b}+\eta_{2 c}^{(2)} \mathcal{P}_{2}^{(2) b}+Q_{c}^{(2)} \lambda^{(2) b}+\right. \\
& \left.\eta_{1 c}^{(2) i} \mathcal{P}_{1 i}^{(2) b}+\eta_{2 c}^{(2) i} \mathcal{P}_{2 i}^{(2) b}+Q_{c}^{(2) i} \lambda_{i}^{(2) b}\right) \times \\
& \times f^{a}{ }_{d e}\left(\eta_{1}^{(2) d} \mathcal{P}_{1}^{(2) e}+\eta_{2}^{(2) d} \mathcal{P}_{2}^{(2) e}+Q^{(2) d} \lambda^{(2) e}+\right. \\
& \left.\eta_{1}^{(2) d i} \mathcal{P}_{1 i}^{(2) e}+\eta_{2}^{(2) d i} \mathcal{P}_{2 i}^{(2) e}+Q^{(2) d i} \lambda_{i}^{(2) e}\right)
\end{aligned}
$$

In order to obtain the path integral of the irreducible model, we work with the gauge-fixing fermion

$$
\begin{aligned}
& K^{T}=\int d^{3} x\left(\mathcal{P}_{1 i}^{(1) a}\left(\epsilon^{0 i j k} \partial_{j} B_{0 k a}+\partial^{i} \varphi_{a}^{(1)}\right)-\frac{1}{2} \mathcal{P}_{1}^{(1) a} \epsilon^{0 i j k} \partial_{i} B_{j k a}+\lambda_{i}^{(1) a} \partial^{i} \eta_{2 a}^{(1)}+\right. \\
& \left.\frac{1}{2} \partial_{[i} \lambda_{j]}^{(1) a}\left(D^{[i}\right)_{a}^{b} \eta_{2 b}^{(2) j]}+f_{b c}^{a} \epsilon^{0 i j k} \pi^{b} \eta_{2 i a}^{(2)} \partial_{j} \lambda_{k}^{(1) c}-\lambda^{(1) a} \partial_{i} \eta_{2 a}^{(1) i}\right) .
\end{aligned}
$$

If we compute the path integral with the help of the above gauge-fixing fermion, we find that its exponent contains a quadratic term, namely,

$$
\begin{aligned}
& \exp \left(i \int d ^ { 4 } x \left(-\frac{1}{2}\left(\left(D_{i}\right)_{a}{ }^{b} B_{b}^{0 i}-f_{a b}^{c}\left(\varphi_{c} \pi^{b}+\varphi_{c}^{(1)} \pi^{b}+\varphi_{c}^{(2)} \pi^{(2) b}\right)-\right.\right.\right. \\
& f^{c}{ }_{a b}\left(\eta_{1 c}^{(2)} \mathcal{P}_{1}^{(2) b}+\eta_{2 c}^{(2)} \mathcal{P}_{2}^{(2) b}+Q_{c}^{(2)} \lambda^{(2) b}+\right. \\
& \left.\left.\left.\left.\eta_{1 c}^{(2) i} \mathcal{P}_{1 i}^{(2) b}+\eta_{2 c}^{(2) i} \mathcal{P}_{2 i}^{(2) b}+Q_{c}^{(2) i} \lambda_{i}^{(2) b}\right)\right)^{2}\right)\right)
\end{aligned}
$$


that can be equivalently written in a linearized form by means of introducing a new field through the Gaussian average

$$
\begin{aligned}
& \int \mathcal{D} H_{0}^{a} \exp \left(i \int d ^ { 4 } x \left(\frac{1}{2} H_{0}^{a} H_{a}^{0}-H_{0}^{a}\left(\left(D_{i}\right)_{a}{ }^{b} B_{b}^{0 i}-\right.\right.\right. \\
& f^{c}{ }_{a b}\left(\varphi_{c} \pi^{b}+\varphi_{c}^{(1)} \pi^{b}+\varphi_{c}^{(2)} \pi^{(2) b}\right)-f^{c}{ }_{a b}\left(\eta_{1 c}^{(2)} \mathcal{P}_{1}^{(2) b}+\right. \\
& \left.\left.\left.\left.\eta_{2 c}^{(2)} \mathcal{P}_{2}^{(2) b}+Q_{c}^{(2)} \lambda^{(2) b}+\eta_{1 c}^{(2) i} \mathcal{P}_{1 i}^{(2) b}+\eta_{2 c}^{(2) i} \mathcal{P}_{2 i}^{(2) b}+Q_{c}^{(2) i} \lambda_{i}^{(2) b}\right)\right)\right)\right)
\end{aligned}
$$

Eliminating some of the auxiliary variables from the resulting gauge-fixed action on their equations of motion, we finally arrive at

$$
\begin{aligned}
& Z_{K^{T}}=\int \mathcal{D} A_{\mu}^{a} \mathcal{D} B_{a}^{\mu \nu} \mathcal{D} \varphi_{a}^{(1)} \mathcal{D} b_{\mu}^{a} \mathcal{D} Q_{a}^{(2) \mu} \mathcal{D} \lambda_{\mu}^{(1) a} \times \\
& \times \mathcal{D} \eta_{1 a}^{(2) \mu} \mathcal{D} \mathcal{P}_{1 \mu}^{(1) a} \mathcal{D} \eta_{2 a}^{(2) \mu} \mathcal{D} \mathcal{P}_{2 \mu}^{(1) a} \exp \left(i S_{K}^{T}\right)
\end{aligned}
$$

where

$$
\begin{aligned}
& S_{K}^{T}=S_{0}^{L}\left[A_{\mu}^{a}, B_{a}^{\mu \nu}\right]+\int d^{4} x\left(b_{\mu}^{a}\left(\frac{1}{2} \epsilon^{\mu \nu \lambda \rho} \partial_{\nu} B_{\lambda \rho a}+\partial^{\mu} \varphi_{a}^{(1)}\right)-\right. \\
& \frac{1}{2} \partial_{[\mu} \lambda_{\nu]}^{(1) a}\left(D^{[\mu}\right)_{a}^{b} Q_{b}^{(2) \nu]}-\left(\partial^{\mu} \lambda_{\mu}^{(1) a}\right)\left(D_{\nu}\right)_{a}{ }^{b} Q_{b}^{(2) \nu}+ \\
& \frac{1}{2} \partial_{[\mu} \mathcal{P}_{1 \nu]}^{(1) a}\left(D^{[\mu}\right)_{a}^{b} \eta_{1 b}^{(2) \nu]}+\left(\partial^{\mu} \mathcal{P}_{1 \mu}^{(1) a}\right)\left(D_{\nu}\right)_{a}{ }^{b} \eta_{1 b}^{(2) \nu}+ \\
& \left.\frac{1}{2} \partial_{[\mu} \mathcal{P}_{2 \nu]}^{(1) a}\left(D^{[\mu}\right)_{a}{ }^{b} \eta_{2 b}^{(2) \nu]}+\left(\partial^{\mu} \mathcal{P}_{2 \mu}^{(1) a}\right)\left(D_{\nu}\right)_{a}{ }^{b} \eta_{2 b}^{(2) \nu}\right),
\end{aligned}
$$

with $S_{0}^{L}\left[A_{\mu}^{a}, B_{a}^{\mu \nu}\right]$ the original action (144). In inferring the above covariant form of the path integral we performed the identifications

$$
\begin{gathered}
A_{\mu}^{a} \equiv\left(H_{0}^{a}, A_{i}^{a}\right), b_{\mu}^{a} \equiv\left(\pi^{(1) a}, \epsilon_{0 i j k} \pi^{j k a}\right), \\
Q_{a}^{(2) \mu} \equiv\left(Q_{a}^{(2)}, Q_{a}^{(2) i}\right), \lambda_{\mu}^{(1) a} \equiv\left(-\lambda^{(1) a}, \lambda_{i}^{(1) a}\right), \\
\eta_{1 a}^{(2) \mu} \equiv\left(-\eta_{1 a}^{(2)}, \eta_{1 a}^{(2) i}\right), \mathcal{P}_{1 \mu}^{(1) a} \equiv\left(\mathcal{P}_{1}^{(1) a}, \mathcal{P}_{1 i}^{(1) a}\right), \\
\eta_{2 a}^{(2) \mu} \equiv\left(-\eta_{2 a}^{(2)}, \eta_{2 a}^{(2) i}\right), \mathcal{P}_{2 \mu}^{(1) a} \equiv\left(\mathcal{P}_{2}^{(1) a}, \mathcal{P}_{2 i}^{(1) a}\right),
\end{gathered}
$$

and, in addition, we adopted the notation

$$
\left(D_{0}\right)_{a}{ }^{b}=\delta_{a}{ }^{b} \partial_{0}-f^{b}{ }_{a c} H_{0}^{c} .
$$

This ends the irreducible BRST-anti-BRST Hamiltonian treatment of the model under study. 


\section{Conclusion}

To conclude with, in this paper we have exposed an irreducible Hamiltonian BRST-anti-BRST method for quantizing reducible first-class systems. The key point of our approach consists in the construction of an irreducible Koszul-Tate bicomplex associated with that of the reducible theory, that reveals some irreducible first-class constraints. Moreover, the physical observables of the starting reducible system and of the resulting irreducible one coincide. This result together with the basic equations of the Hamiltonian BRST-anti-BRST method allow the replacement of the Hamiltonian BRST-anti-BRST quantization of the initial reducible system with that of the irreducible theory. The existence of the canonical generator of the ir-

reducible BRST-anti-BRST symmetry is provided by the biacyclicity of the irreducible Koszul-Tate bicomplex, while the gauge-fixing procedure is facilitated by the enlargement of the phase-space with the canonical pairs of the type $(y, \pi)$. The theoretical part of the paper has been exemplified on the Freedman-Townsend model.

\section{Acknowledgment}

This work has been supported by a Romanian National Council for Academic Scientific Research (CNCSIS) grant.

\section{References}

[1] M. Henneaux and C. Teitelboim, Quantization of Gauge Systems, Princeton Univ. Press, (Princeton, 1992)

[2] I. A. Batalin, P. M. Lavrov and I. V. Tyutin, J. Math. Phys. 31, 6 (1990)

[3] I. A. Batalin, P. M. Lavrov and I. V. Tyutin, J. Math. Phys. 31, 2708 (1990)

[4] Ph. Grégoire and M. Henneaux, Phys. Lett. B277, 459 (1992)

[5] Ph. Grégoire and M. Henneaux, Commun. Math. Phys. 157, 279 (1993) 
[6] Ph. Grégoire, Ph.D. thesis, Université Libre de Bruxelles, 1993

[7] I. A. Batalin, P. M. Lavrov and I. V. Tyutin, J. Math. Phys. 31, 1487 (1990)

[8] I. A. Batalin, P. M. Lavrov and I. V. Tyutin, J. Math.Phys. 32, 532 (1991)

[9] M. Henneaux, Phys. Lett. B282, 372 (1992)

[10] Ph. Grégoire and M. Henneaux, J. Phys. A Math. Gen. 26, 6073 (1993)

[11] J. Hoyos, M. Quiros, J. Ramirez Mittelbrunn and F. J. de Urries, Nucl. Phys. B218, 159 (1983)

[12] V. P. Spiridonov, Nucl. Phys. B308, 257 (1988)

[13] J. Gomis and J. Roca, Nucl. Phys. B342, 152 (1990)

[14] E. Dur and S. J. Gates Jr., Nucl. Phys. B343, 622 (1990)

[15] C. M. Hull, Mod. Phys. Lett. A5, 1871 (1990)

[16] C. M. Hull, B. Spence and J. L. Vasquez-Bello, Nucl. Phys. B348, 108 (1991)

[17] L. Tatar and R. Tatar, Phys. Lett. B326, 238 (1994)

[18] L. Tataru, J. Phys. A: Math. Gen. 28, 4175 (1995)

[19] L. Tataru and R. Tatar, Int. J. Mod. Phys. A13, 1981 (1998)

[20] L. Bonora and M. Tonin, Phys. Lett. B98, 83 (1981)

[21] L. Bonora, P. Pasti and M. Tonin, J. Math. Phys. 23, 83 (1982)

[22] L. Baulieu and J. Thierry-Mieg, Nucl. Phys. B197, 477 (1982)

[23] F. R. Ore and P. van Nieuwenhuizen, Nucl. Phys. B204, 317 (1982)

[24] L. Alvarez-Gaumé and L. Baulieu, Nucl. Phys. B212, 255 (1983)

[25] S. Hwang, Nucl. Phys. B231, 386 (1984) 
[26] S. Hwang, Nucl. Phys. B322, 107 (1989)

[27] R. Banerjee and J. Barcelos-Neto, Ann. Phys. (N.Y.) 265, 134 (1998)

[28] C. Bizdadea and S. O. Saliu, J. Phys. A: Math. Gen. 31, 8805 (1998); 32, 3919 (1999)

[29] C. Bizdadea, I. Negru and S. O. Saliu, Int. J. Mod. Phys. A14, 359 (1999)

[30] P. A. M. Dirac, Lectures on Quantum Mechanics, Yeshiva University (New York: Academic Press, 1967)

[31] D. Z. Freedman and P. K. Townsend, Nucl. Phys. B177, 282 (1981) 